\title{
Singularity-free Design of the Translational Parallel Manipulator IRSBot-2
}

\author{
Coralie Germain Stéphane Caro* Sébastien Briot Philippe Wenger \\ Institut de Recherche en Communications et Cybernétique de Nantes \\ UMR CNRS nº 6597 \\ 1 rue de la Noë, 44321 Nantes, France \\ Emails: germain, caro, briot, wenger@irccyn.ec-nantes.fr
}

\begin{abstract}
The IRSBot-2 is a two degree-of-freedom translational parallel manipulator dedicated to fast and accurate pick-and-place operations. This paper deals with the determination of the design parameters of the manipulator for the IRSBot-2 to be free of parallel singularity. First, the robot architecture is introduced. The IRSBot-2 is composed of two identical spatial limbs, each one containing a proximal module and a distal module. Then, its actuation singularities and constraint singularities are analyzed. The latter are analyzed in its distal parameter space with a method based on the notion of Discriminant Varieties and Cylindrical Algebraic Decomposition. Moreover, a deep analysis is carried out in order to determine the set of design parameters of the distal modules that prevents the IRSBot- 2 from reaching any constraint singularity. To the best of our knowledge, such an analysis is performed for the first time. Finally, a design methodology is proposed to determine the set of design parameters associated with the proximal modules for the IRSBot-2 to be assembled and free of parallel singularity.
\end{abstract}

Keywords: Parallel Manipulator; Constraint Singularity; Actuation Singularity; Cylindrical Algebraic Decomposition; Design.

\section{Introduction}

Several robot architectures for high-speed operations have been proposed in the past decades $[7,4,14$, 19, 16]. Many of them have four degrees of freedom (dof): three translations and one rotation about a fixed axis (Schoenflies motions $[6,15]$ ). Some simple operations need only two translational dof in order to move a part from a working area to another. Therefore, several robot architectures with two

\footnotetext{
${ }^{*}$ Corresponding author: Stéphane Caro; Email: stephane.caro@irccyn.ec-nantes.fr; Tel: +33 2403769 68; Fax: +33240376930
} 
translational dof have been proposed. Among them, those that have the capacity to fix the orientation of the platform via the use of a planar parallelogram (also called a $\Pi$ joint) are necessary in numerous operations. For example, Brogårdh proposed in [5] an architecture made of a $\Pi$ joint located between the linear actuators and the platform. Another 2-dof translational robot was presented in [16], where the authors use two $\Pi$ joints to link the platform with two vertical prismatic actuators. Its equivalent architecture actuated by revolute joints is presented in [11].

The main common point between these architectures is that they are all planar, i.e. all their elements are constrained to move in the plane of motion. As a result, all their elements are subject to bending effects in the direction normal to the plane of motion. In order to guarantee a minimum stiffness in this direction, the elements have to be bulky, leading to high inertia and to low acceleration capacities. In order to overcome these problems, a new Delta-like robot, named the Par2, was proposed in [20]. The Par2 has the following properties: all the elements of the distal parts of the legs are only subject to tension/compression effects. This leads to a lighter structure with better acceleration capacities. The authors successfully built a prototype that can reach $53 \mathrm{G}$. However, even if its acceleration capacities are impressive, its accuracy is poor. This phenomenon can be explained by the complexity of the architecture composed of four identical legs among which two of them are linked with a rigid belt. As a result, this robot is more subject to parasitic effects that are difficult to identify and can decrease its accuracy. Moreover, its Cartesian workspace is rather small because the robot has four legs.

A two-dof spatial translational robot, named IRSBot-2, was introduced in [10] to overcome its counterparts in terms of mass in motion, stiffness and workspace size. IRSBot-2 stands for "IRCCyN Spatial Robot with 2 dof". The IRSBot-2 has a spatial architecture and the distal parts of its legs are subject only to tension/compression/torsion. As a result, its stiffness is increased and its total mass can be reduced. It is composed of two legs only in order to reduce the mechanism complexity and to increase the size of its Cartesian workspace.

The advantages of the IRSBot2 in terms of stiffness and weight reduction were disclosed in [10]. It was shown that this robot is lighter than the Par2 and the five-bar mechanism while being stiffer than the latter.

Nevertheless, the IRSBot-2 may reach some constraint singularities like many lower-mobiliy parallel manipulators [21, 2]. In this paper, a deep analysis is carried out in order to determine the sets of design parameters of the IRSBot- 2 that prevent it from reaching any constraint singularity. To the best of our knowledge, such an analysis is performed for the first time and is very helpful for the robot designer. Indeed, it allows the designer to select the design parameters of the manipulator in such a way that the manipulator cannot reach any parallel singularity.

This paper is organized as follows. First, the robot architecture is described and its constraint singularity conditions are derived. Then, its constraint singularities are analyzed in its parameter space based on a cylindrical algebraic decomposition. Moreover, the set of design parameters for the robot to be free of constraint singularity are determined. Finally, a design methodology is proposed to determine the set of design parameters associated with the proximal modules for the IRSBot- 2 to be assembled and free of singularity. 


\section{Robot Architecture}

The IRSBot-2 has two translational degrees of freedom along the $\mathbf{x}_{0}$ and $\mathbf{z}_{0}$ axes of the robot base frame as shown in Fig. 1. It is composed of two identical legs connecting the fixed base to the moving platform.

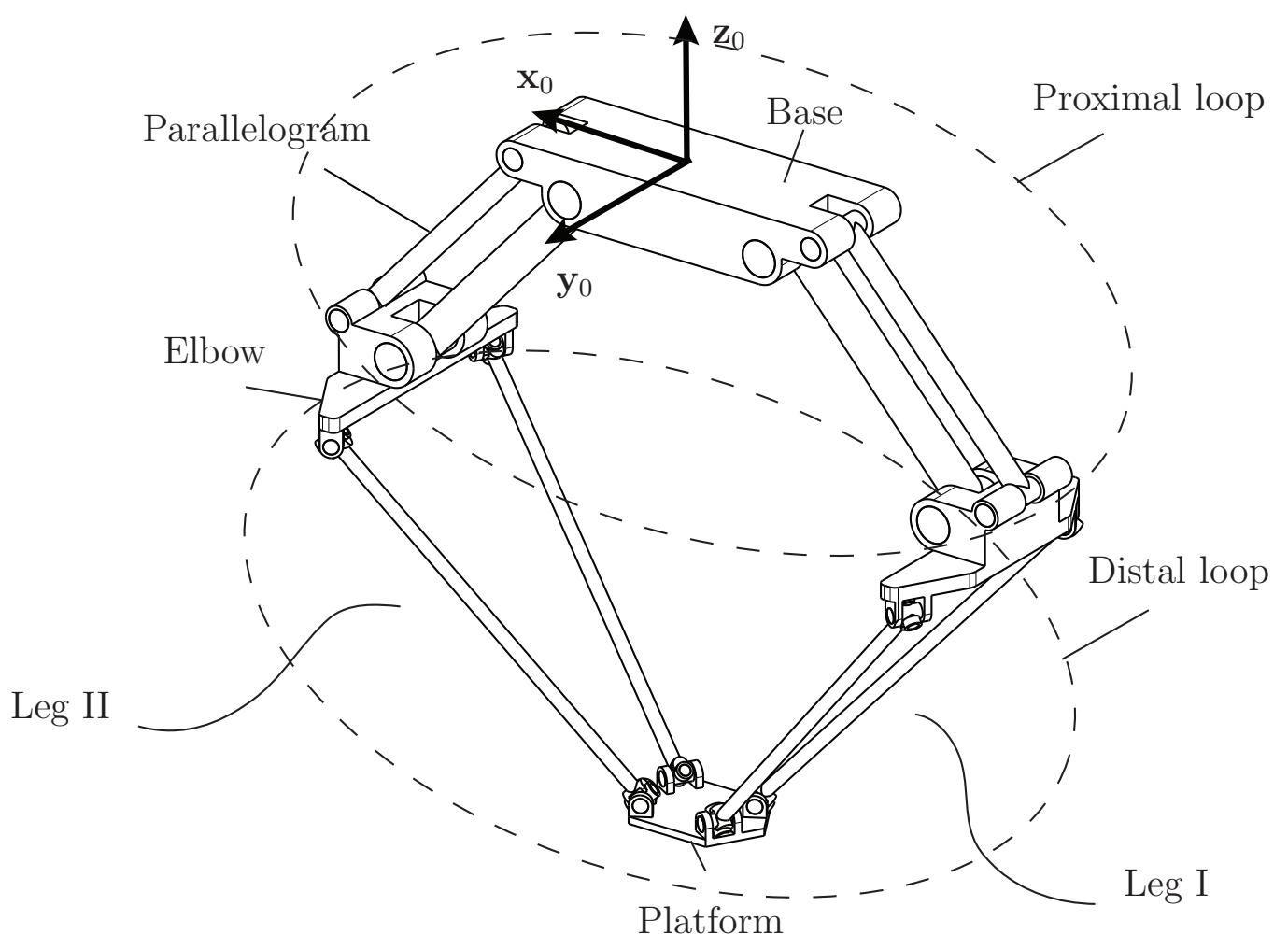

Figure 1: CAD Modeling of the IRSBot-2

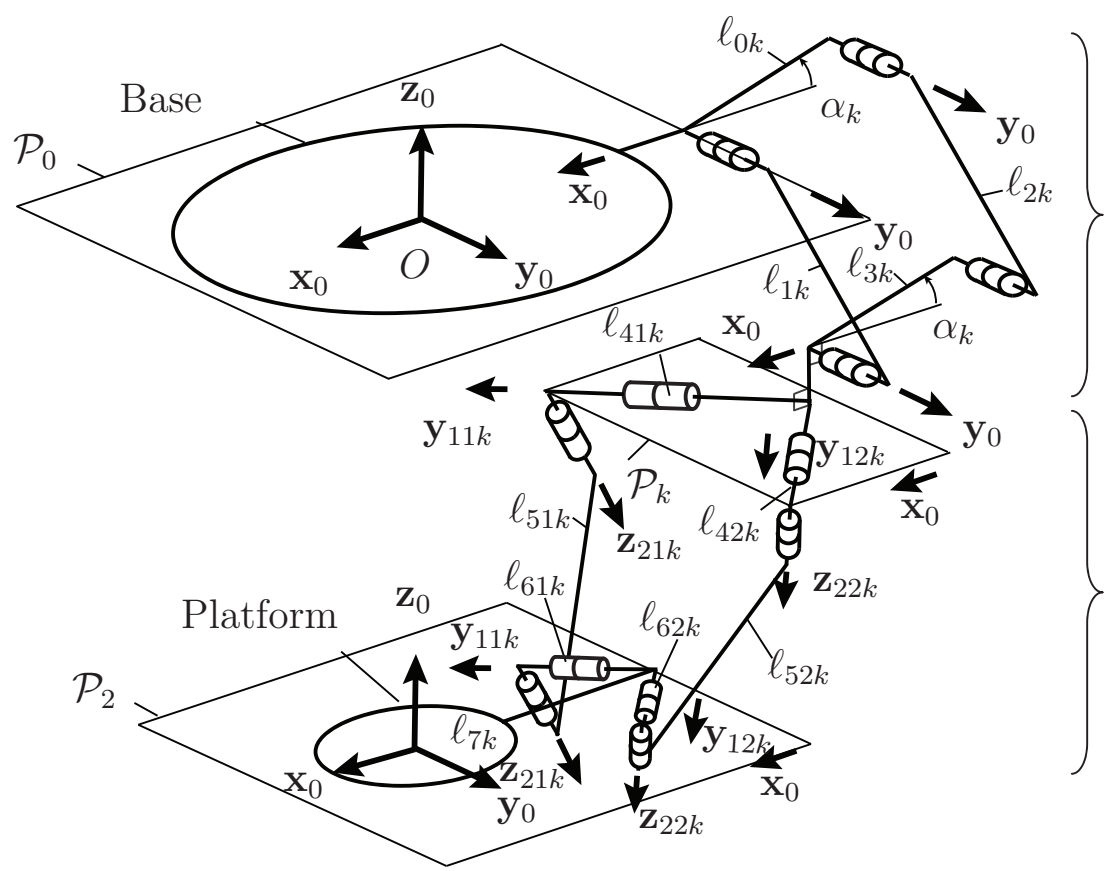

Proximal module

Distal module

Figure 2: Kinematic chain of the $k$ th $\operatorname{leg}(k=I, I I)$ 
The $k$ th leg of the IRSBot- 2 is described in Fig. 2 and contains one proximal module and one distal module $(k=I, I I)$. Therefore, the IRSBot-2 has one proximal loop and one distal loop shown in Fig. 1. The former is composed of the two proximal modules and the base. The latter is composed of the two distal modules and the moving-platform.

On the one hand, the proximal module amounts to a $\Pi$ joint of normal $\mathbf{y}_{0}$ and is made up of links $\ell_{0 k}, \ell_{1 k}, \ell_{2 k}$ and $\ell_{3 k}$. The proximal module aims to keep planes $\mathcal{P}_{0}$ and $\mathcal{P}_{k}$ parallel. The frame $\left(\mathbf{O}, \mathbf{x}_{\mathbf{0}}, \mathbf{y}_{\mathbf{0}}, \mathbf{z}_{\mathbf{0}}\right)$ is attached to plane $\mathcal{P}_{0}$.

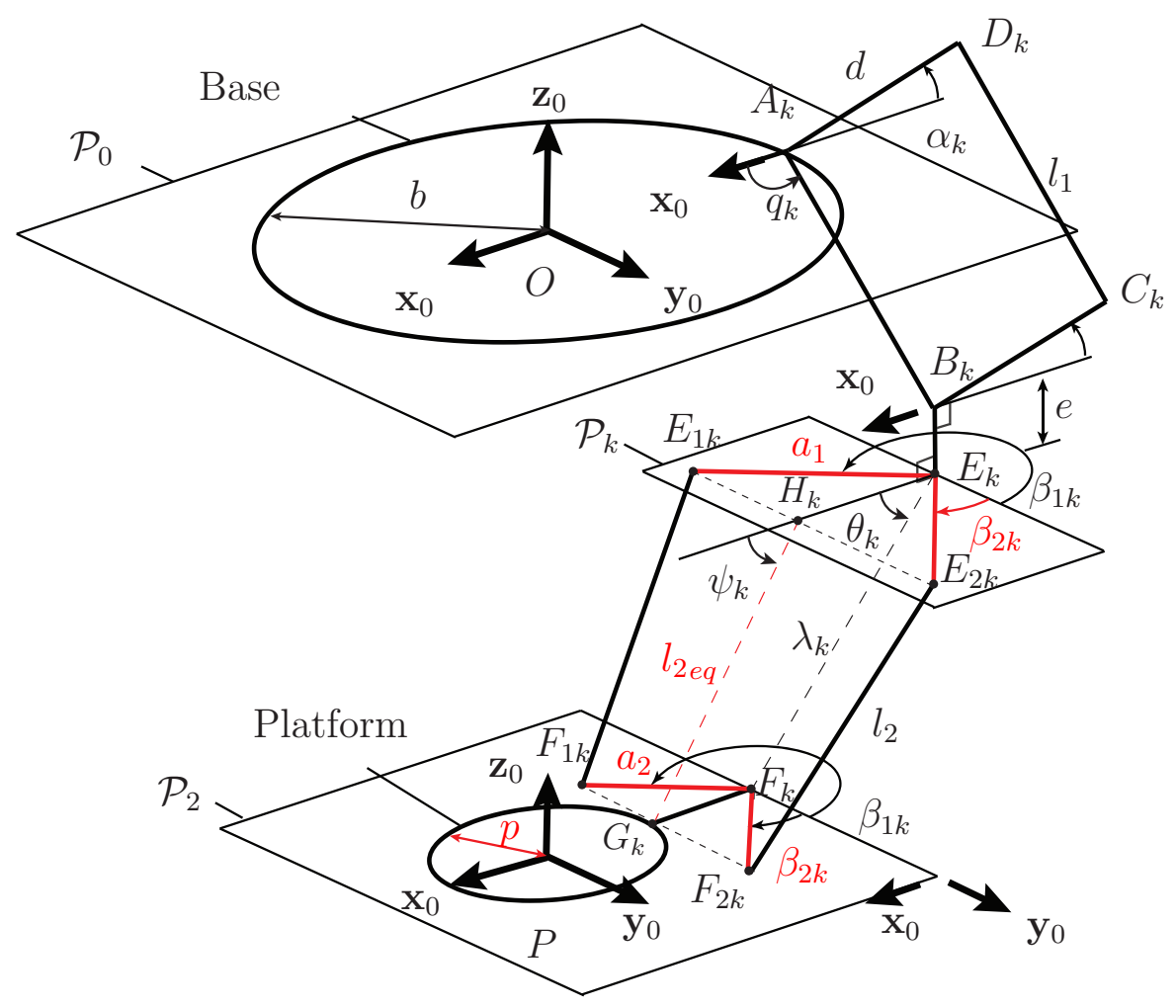

Figure 3: Paramaterization of the $k$ th leg $(k=I, I I)$

On the other hand, the distal module is attached to link $\ell_{3 k}$ of the parallelogram through two revolute joints of axis $\left(E_{k}, \mathbf{y}_{1 j k}\right)$ lying in plane $\mathcal{P}_{k}$ and to link $\ell_{7 k}$ of the moving platform through two revolute joints of axis $\left(F_{k}, \mathbf{y}_{1 j k}\right)$ lying in plane $\mathcal{P}_{2}(j=1,2)$. Axes $\mathbf{y}_{11 k}$ and $\mathbf{y}_{12 k}\left(\mathbf{z}_{21 k}\right.$ and $\mathbf{z}_{22 k}$, resp.) are symmetrical with respect to plane $\left(\mathbf{x}_{0} O \mathbf{z}_{0}\right)$. It should be mentioned that axes $\mathbf{y}_{1 j k}$ and $\mathbf{z}_{2 j k}$ are orthogonal and have to be both orthogonal to link $\ell_{5 j k}$. Links $\ell_{51 k}$ and $\ell_{52 k}$ (links $\ell_{41 k}$ and $\ell_{42 k}$, resp.) are not parallel, otherwise the distal module would become a spatial parallelogram and the robot architecture would be singular. The distal module may be decomposed into two identical parts composed of links $\ell_{4 j k}, \ell_{5 j k}$ and $\ell_{6 j k}$, which are linked together with revolute joints of axes $\mathbf{z}_{2 j k}$. The robot is assembled in such a way that planes $\mathcal{P}_{k}$ and $\mathcal{P}_{2}$ remain parallel. Therefore, $\mathcal{P}_{2}$ is also parallel to $\mathcal{P}_{0}$.

The design parameters of the IRSBot-2 are depicted in Figs. 3 and 4. The parameters used to define the kinematic model of the IRSBot-2 robot are depicted in Fig. 3 [10]. $q_{k}$ is the actuated joint coordinate of the $k$ th leg, $b=O A_{k}$ is the radius of the base, $l_{1}=A_{k} B_{k}$ is the length of the proximal legs, $l_{2}=E_{j k} F_{j k}$ is the length of the spatial distal legs, $a_{1}$ and $a_{2}$ denote the lengths of segments 


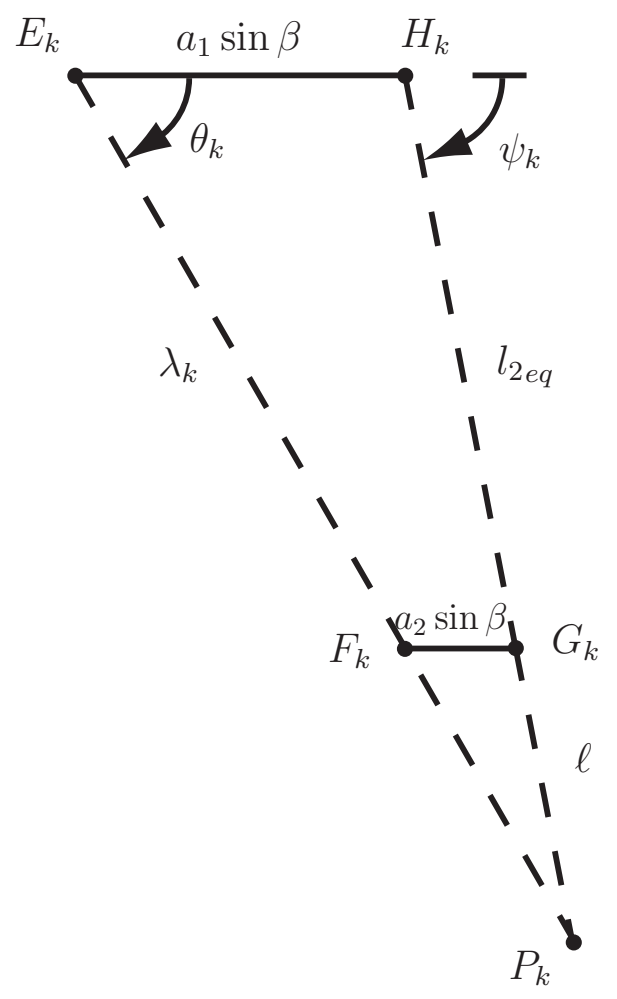

Figure 4: Closed-loop $E_{k}-H_{k}-G_{k}-F_{k}$ : projection of the distal module on the plane $\left(\mathbf{x}_{0} O \mathbf{z}_{0}\right)$

$E_{k} E_{j k}$ and $F_{k} F_{j k}$. One can notice that the angle between $\mathbf{y}_{0}$ and $E_{k} E_{j k}$ (resp. $\mathbf{y}_{0}$ and $F_{k} F_{j k}$ ) is constant and equal to $\beta_{j k}$. Let $\beta$ denote $\beta_{2 I I}$, then $\beta_{1 I}=\pi+\beta, \beta_{2 I}=-\beta$ and $\beta_{1 I I}=\pi-\beta$. Angle $\beta$ is strictly bounded between 0 and $\pi / 2$, i.e., $0<\beta<\pi / 2$, as links $\ell_{41 k}$ and $\ell_{42 k}$ can not be parallel.

Points $H_{k}$ and $G_{k}$ are the midpoints of segments $E_{1 k} E_{2 k}$ and $F_{1 k} F_{2 k}$, respectively. By construction, $H_{k}$ lies in plane $\mathcal{P}_{1}$ and $G_{k}$ lies in plane $\mathcal{P}_{2}$. From the Pythagorean theorem, the length between points $H_{k}$ and $G_{k}$ is constant and equal to:

$$
l_{2 e q}=\sqrt{l_{2}^{2}-\left(a_{1}-a_{2}\right)^{2} \cos ^{2} \beta}
$$

$\lambda_{k}$ is the distance between points $E_{k}$ and $F_{k}$. Let $\psi_{k}$ be the angle between $\mathbf{x}_{0}$ and $\overrightarrow{H_{k} G_{k}}$ and $\theta_{k}$ be the angle between $\mathbf{x}_{0}$ and $\overrightarrow{E_{k} F_{k}}$. Finally, $p=P G_{k}$ is the radius of the moving platform and $e$ is an offset along $\mathbf{z}_{0}$ between the proximal and distal modules.

\section{Constraint Analysis of the IRSBot-2}

In this section, a constraint analysis of the IRSBot- 2 is carried out by using the reciprocal screw theory $[9,12,13]$ in order to determine its constraint wrench system $\mathcal{W}_{I R S}^{c}$ and actuation wrench system $\mathcal{W}_{I R S}^{a}$ and to analyze its parallel singularities, namely, its constraint and actuation singularities. On the one hand, the IRSBot-2 may reach some constraint singularities as it is a lower-mobility parallel manipulator. As a result, the system of output freedoms instantaneously increases its dimension. The extra degree of freedom of the platform may not be controllable by the actuators in such configu- 
rations [21]. The IRSBot-2 reaches a constraint singularity when $\mathcal{W}_{I R S}^{c}$ degenerates. On the other hand, the IRSBot-2 meets an actuation singularity when the system spanned by $\mathcal{W}_{I R S}^{c}$ and $\mathcal{W}_{I R S}^{a}$ degenerates, whereas $\mathcal{W}_{I R S}^{c}$ does not $[3,1]$.

\subsection{Constraint wrench system of the IRSBot-2}

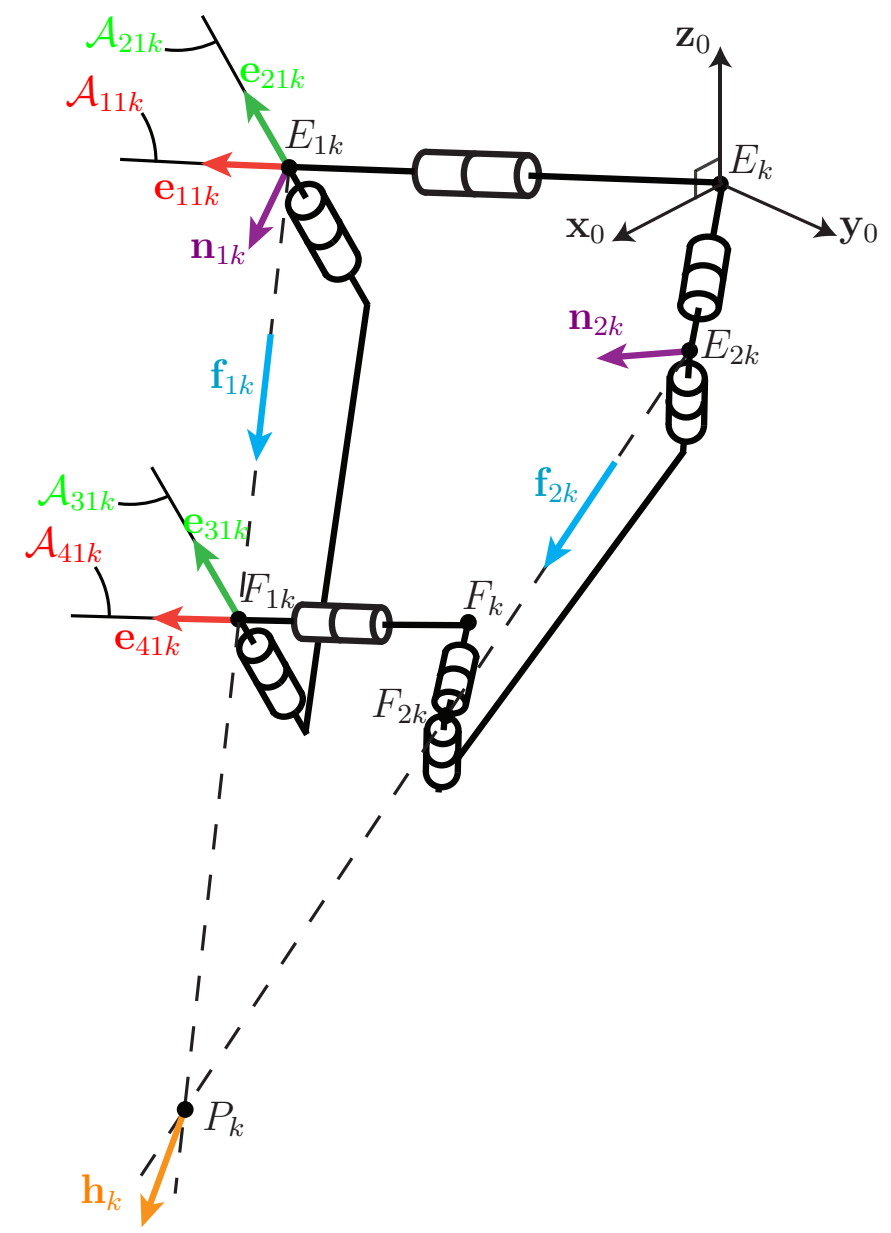

Figure 5: Kinematic chain of the $k$ th distal module of the IRSBot-2 $(k=I, I I)$

Figure 5 represents the distal module of the $k$ th leg of the IRSBot-2 $(k=I, I I)$. This distal module is composed of two symmetrical serial kinematic chains $\mathcal{L}_{j k}: E_{k}-E_{j k}-F_{j k}-F_{k}(j=1,2)$, each one containing four revolute joints of axes $\mathcal{A}_{1 j k}, \mathcal{A}_{2 j k}, \mathcal{A}_{3 j k}$ and $\mathcal{A}_{4 j k}$, respectively. Let $\mathbf{e}_{i j k}$ be the unit vector of axis $\mathcal{A}_{i j k},(i=1, \ldots, 4)$. The following geometric conditions appear: $(i)$ axes $\mathcal{A}_{i 1 k}$ and $\mathcal{A}_{i 2 k}$ are symmetrical with respect to plane $\left(\mathbf{x}_{0} O \mathbf{z}_{0}\right) ;(i i)$ axes $\mathcal{A}_{1 j k}$ and $\mathcal{A}_{2 j k}$ intersect at point $E_{j k}$; (i i i ) axes $\mathcal{A}_{3 j k}$ and $\mathcal{A}_{4 j k}$ intersect at point $F_{j k}$; (iv) axes $\mathcal{A}_{2 j k}$ and $\mathcal{A}_{3 j k}$ are parallel:

$$
\mathbf{e}_{2 j k}=\mathbf{e}_{3 j k}
$$

Besides, axes $\mathcal{A}_{1 j k}$ and $\mathcal{A}_{4 j k}$ turn to be parallel when the IRSBot-2 is assembled, namely,

$$
\mathbf{e}_{1 j k}=\mathbf{e}_{4 j k}
$$


The serial kinematic chain $E_{k}-E_{j k}-F_{j k}-F_{k}$ contains four revolute joints. Therefore, its twist-system is spanned by four zero-pitch twists ${ }^{1}$, namely,

$$
\mathcal{T}_{j k}=\operatorname{span}\left(\hat{\mathcal{E}}_{01 j k}, \hat{\mathcal{E}}_{02 j k}, \hat{\mathcal{E}}_{03 j k}, \hat{\mathcal{E}}_{04 j k}\right)
$$

where

$$
\begin{aligned}
& \hat{\mathcal{E}}_{01 j k}=\left(\mathbf{e}_{1 j k}, \mathbf{r}_{E j k} \times \mathbf{e}_{1 j k}\right) \\
& \hat{\mathcal{E}}_{02 j k}=\left(\mathbf{e}_{2 j k}, \mathbf{r}_{E j k} \times \mathbf{e}_{2 j k}\right) \\
& \hat{\mathcal{E}}_{03 j k}=\left(\mathbf{e}_{3 j k}, \mathbf{r}_{F j k} \times \mathbf{e}_{3 j k}\right) \\
& \hat{\mathcal{E}}_{04 j k}=\left(\mathbf{e}_{4 j k}, \mathbf{r}_{F j k} \times \mathbf{e}_{4 j k}\right)
\end{aligned}
$$

$\mathbf{r}_{E j k}$ and $\mathbf{r}_{F j k}$ denote the position vectors of points $E_{j k}$ and $F_{j k}$, respectively.

The constraint wrench system $\mathcal{W}_{j k}$ of $\mathcal{L}_{j k}$ contains wrenches that are reciprocal to twists $\hat{\mathcal{E}}_{01 j k}$, $\hat{\mathcal{E}}_{02 j k}, \hat{\mathcal{E}}_{03 j k}$ and $\hat{\mathcal{E}}_{04 j k}$, namely a 2-sytem given by:

$$
\mathcal{W}_{j k}=\operatorname{span}\left(\hat{\mathcal{F}}_{j k}, \hat{\mathcal{M}}_{j k}\right)
$$

where

$$
\begin{aligned}
\hat{\mathcal{F}}_{j k} & =\left(\mathbf{f}_{j k}, \mathbf{r}_{F j k} \times \mathbf{f}_{j k}\right) \\
\hat{\mathcal{M}}_{j k} & =\left(\mathbf{0}_{3 \times 1}, \mathbf{n}_{j k}\right)
\end{aligned}
$$

$\mathbf{f}_{j k}$ is the unit vector of $\overrightarrow{E_{j k} F_{j k}}$ and $\mathbf{n}_{j k}=\mathbf{e}_{1 j k} \times \mathbf{e}_{2 j k}=\mathbf{e}_{3 j k} \times \mathbf{e}_{4 j k}$.

Therefore, the constraint wrench system $\mathcal{W}_{k}$ of the distal module of the $k$ th leg takes the form:

$$
\mathcal{W}_{k}=\operatorname{span}\left(\hat{\mathcal{F}}_{1 k}, \hat{\mathcal{F}}_{2 k}, \hat{\mathcal{M}}_{1 k}, \hat{\mathcal{M}}_{2 k}\right)
$$

The twist system $\mathcal{T}_{k}$ of the distal module of the $k$ th leg can be derived from Eq. (12). As a matter of fact, it is a 2 -system and contains twists that are reciprocal to wrenches $\hat{\mathcal{F}}_{1 k}, \hat{\mathcal{F}}_{2 k}, \hat{\mathcal{M}}_{1 k}$ and $\hat{\mathcal{M}}_{2 k}$ :

$$
\mathcal{T}_{k}=\operatorname{span}\left(\hat{\mathcal{E}}_{0 k}, \hat{\mathcal{E}}_{\infty k}\right)
$$

where

$$
\begin{aligned}
\hat{\mathcal{E}}_{0 k} & =\left(\mathbf{n}_{1 k} \times \mathbf{n}_{2 k}, \mathbf{r}_{P k} \times\left(\mathbf{n}_{1 k} \times \mathbf{n}_{2 k}\right)\right) \\
\hat{\mathcal{E}}_{\infty k} & =\left(\mathbf{0}_{3 \times 1}, \mathbf{f}_{1 k} \times \mathbf{f}_{2 k}\right)
\end{aligned}
$$

$\mathbf{r}_{P k}$ denotes the position vector of point $P_{k}$, which is the intersection point of lines $\left(E_{1 k} F_{1 k}\right)$ and $\left(E_{2 k} F_{2 k}\right)$ as shown in Fig. 5.

\footnotetext{
${ }^{1}$ A zero-pitch twist $\hat{\mathcal{E}}_{0}=\left(\mathbf{u}, \mathbf{r}_{A} \times \mathbf{u}\right)$ corresponds to a pure rotation about an axis of unit vector $\mathbf{u}$ and passing through point $A$. An infinite-pitch twist $\hat{\mathcal{E}}_{\infty}=\left(\mathbf{0}_{3 \times 1}, \mathbf{v}\right)$ corresponds to a pure translation along a direction of unit vector $\mathbf{v}$.
} 
Let $\hat{\mathcal{E}}_{\infty p k}$ be the twist associated with the proximal module of the $k$ th leg of the IRSBot-2:

$$
\hat{\mathcal{E}}_{\infty p k}=\left(\mathbf{0}_{3 \times 1}, \mathbf{y}_{0} \times \mathbf{g}_{k}\right)
$$

where $\mathbf{g}_{k}$ is the unit vector of line $\left(A_{k} B_{k}\right)$ as depicted in Fig. 6.

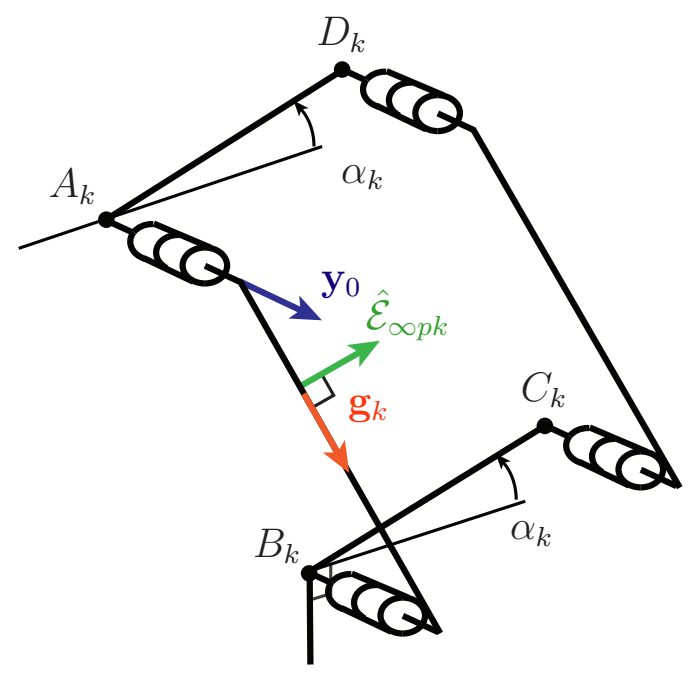

Figure 6: Kinematic chain of the $k$ th proximal module of the IRSBot-2 $(k=I, I I)$

Accordingly, the twist system $\mathcal{T}_{\text {Legk }}$ of the $k$ th leg of the IRSBot- 2 is spanned by one zero-pitch twist and two-infinite pitch twists:

$$
\mathcal{T}_{\text {Legk }}=\operatorname{span}\left(\hat{\mathcal{E}}_{0 k}, \hat{\mathcal{E}}_{\infty k}, \hat{\mathcal{E}}_{\infty p k}\right)
$$

It means that the $k$ th leg of the IRSBot-2 amounts to a serial kinematic chain composed of one revolute joint and two prismatic joints. The axis $\mathcal{A}_{k}$ of the corresponding revolute joint passes through point $P_{k}$ shown in Fig. 5 and is along the unit vector $\mathbf{n}_{1 k} \times \mathbf{n}_{2 k}$. The directions of the two prismatic joints are along the unit vectors $\mathbf{f}_{1 k} \times \mathbf{f}_{2 k}$ and $\mathbf{y}_{0} \times \mathbf{g}_{k}$, respectively. Note that the three vectors $\mathbf{n}_{1 k} \times \mathbf{n}_{2 k}$, $\mathbf{f}_{1 k} \times \mathbf{f}_{2 k}$ and $\mathbf{y}_{0} \times \mathbf{g}_{k}$ are normal to vector $\mathbf{y}_{0}$ while points $P_{I}$ and $P_{I I}$ lie in the plane $\left(\mathbf{x}_{0} O \mathbf{z}_{0}\right)$. As a consequence, Fig. 7 illustrates an instantaneous planar equivalent closed kinematic chain of the IRSBot-2.

The constraint wrench system $\mathcal{W}_{\text {Legk }}$ of the $k$ th leg of the IRSBot-2 is reciprocal to twists $\hat{\mathcal{E}}_{0 k}$, $\hat{\mathcal{E}}_{\infty k}$ and $\hat{\mathcal{E}}_{\infty p k}$ :

$$
\mathcal{W}_{\text {Legk }}=\mathcal{T}_{\text {Legk }}^{\perp}
$$

Thus, it is a three-system spanned by the two moments $\hat{\mathcal{M}}_{1 k}, \hat{\mathcal{M}}_{2 k}$ expressed in Eq. (11) and the pure force $\hat{\mathcal{F}}_{k}$ defined as follows:

$$
\hat{\mathcal{F}}_{k}=\left(\mathbf{y}_{0}, \mathbf{r}_{M k} \times \mathbf{y}_{0}\right)
$$

$\mathbf{r}_{M k}$ denotes the position vector of any point $M_{k}$ on the axis $\mathcal{A}_{k}$ of unit vector $\mathbf{n}_{1 k} \times \mathbf{n}_{2 k}$ and passing through point $P_{k}$ shown in Fig. 7. 


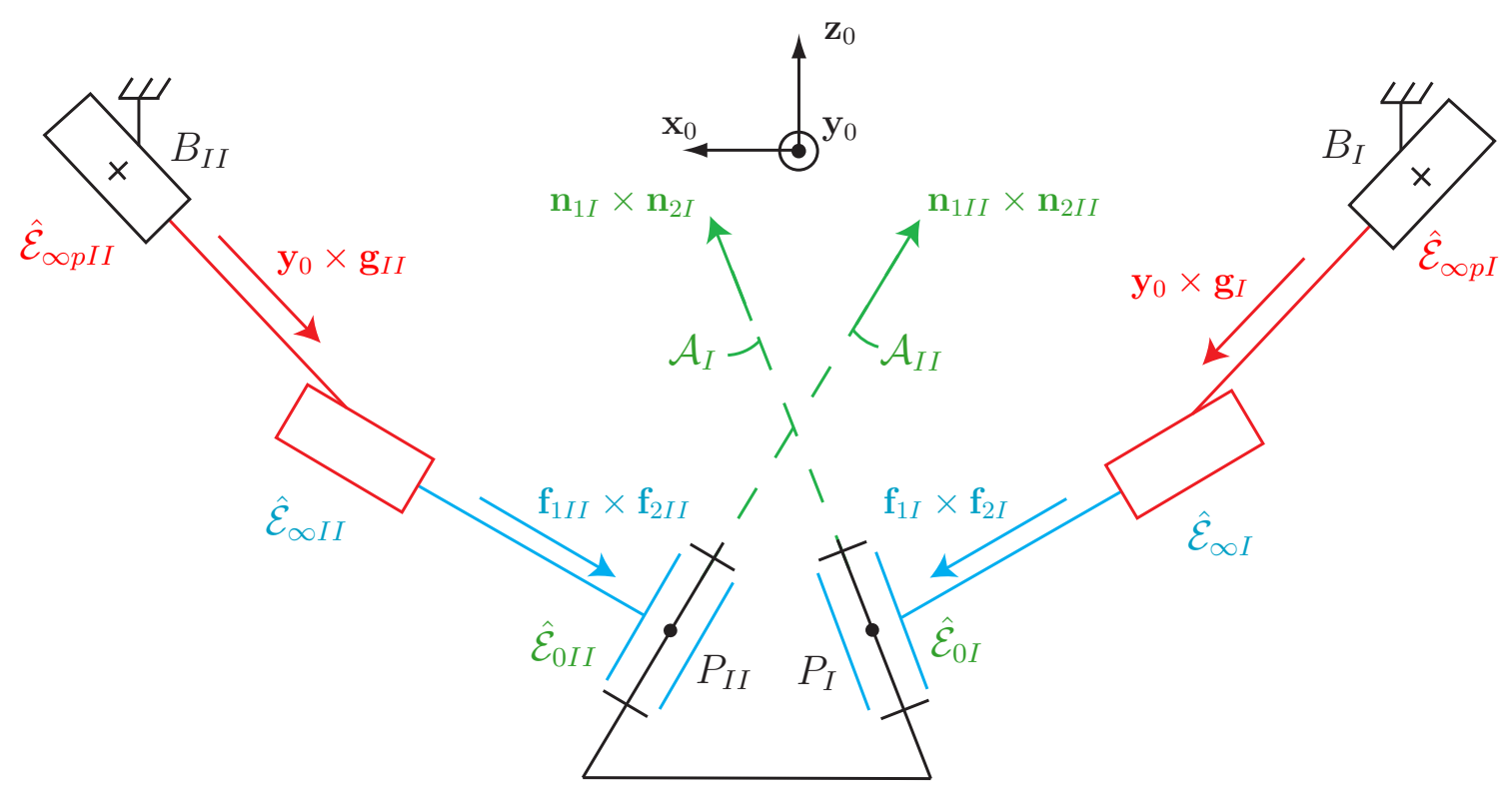

Figure 7: Equivalent instantaneous mechanism of the IRSBot-2

As a result, the constraint wrench system $\mathcal{W}_{I R S}^{c}$ of the IRSBot2 is expressed as follows:

$$
\begin{aligned}
\mathcal{W}_{I R S}^{c} & =\mathcal{W}_{\text {LegI }}+\mathcal{W}_{\text {LegII }} \\
& =\operatorname{span}\left(\hat{\mathcal{M}}_{1 I}, \hat{\mathcal{M}}_{2 I}, \hat{\mathcal{F}}_{I}, \hat{\mathcal{M}}_{1 I I}, \hat{\mathcal{M}}_{2 I I}, \hat{\mathcal{F}}_{I I}\right)
\end{aligned}
$$

As the axes of the pure forces $\hat{\mathcal{F}}_{I}$ and $\hat{\mathcal{F}}_{I I}$ are parallel, span $\left(\hat{\mathcal{F}}_{I}, \hat{\mathcal{F}}_{I I}\right)=\operatorname{span}\left(\hat{\mathcal{F}}_{I}, \hat{\mathcal{M}}_{I I I}\right)$ with

$$
\hat{\mathcal{M}}_{I I I}=\left(\mathbf{0}_{3 \times 1}, \mathbf{y}_{0} \times \overrightarrow{M_{I} M_{I I}}\right)
$$

$\mathcal{W}_{I R S}^{c}$ can also take the form:

$$
\mathcal{W}_{I R S}^{c}=\operatorname{span}\left(\hat{\mathcal{M}}_{1 I}, \hat{\mathcal{M}}_{2 I}, \hat{\mathcal{M}}_{1 I I}, \hat{\mathcal{M}}_{2 I I}, \hat{\mathcal{F}}_{I}, \hat{\mathcal{M}}_{I I I}\right)
$$

It is noteworthy that $\mathcal{W}_{I R S}^{c}$ is spanned by one pure force $\hat{\mathcal{F}}_{I}$ and five pure moments $\hat{\mathcal{M}}_{1 I}, \hat{\mathcal{M}}_{2 I}, \hat{\mathcal{M}}_{1 I I}$, $\hat{\mathcal{M}}_{2 I I}$ and $\hat{\mathcal{M}}_{I I I}$.

\subsection{Twist system of the IRSBot-2}

The twist system $\mathcal{T}_{I R S}$ of the IRSBot-2 is reciprocal to its global constraint wrench system $\mathcal{W}_{I R S}^{c}$ expressed in Eq. (23), namely, it is spanned by two infinite pitch twists of directions normal to vector $\mathbf{y}_{0}$ :

$$
\mathcal{T}_{I R S}=\operatorname{span}\left(\hat{\mathcal{E}}_{\infty \mathbf{x}_{0}}, \hat{\mathcal{E}}_{\infty \mathbf{z}_{0}}\right)
$$


with

$$
\begin{aligned}
& \hat{\mathcal{E}}_{\infty \mathbf{x}_{0}}=\left(\mathbf{0}_{3 \times 1}, \mathbf{x}_{0}\right) \\
& \hat{\mathcal{E}}_{\infty \mathrm{z}_{0}}=\left(\mathbf{0}_{3 \times 1}, \mathbf{z}_{0}\right)
\end{aligned}
$$

Practically, in the assembly mode of the manipulator under study, the moving platform of the IRSBot-2 can perform two-dof translational motions in the plane $\left(\mathbf{x}_{0} O \mathbf{z}_{0}\right)$.

\subsection{Actuation wrench system of the IRSBot-2}

Let us consider that the actuated joint of a leg of the IRSBot-2 is locked and let $\mathcal{U}_{\text {Legk }}$ denote the wrench system reciprocal to the unactuated joints of the leg. Generally,

$$
\operatorname{dim}\left(\mathcal{U}_{\text {Legk }}\right)=\operatorname{dim}\left(\mathcal{W}_{\text {Legk }}\right)+1
$$

Then, $\mathcal{U}_{\text {Legk }}$ includes $\mathcal{W}_{\text {Legk }}$ plus a set of some additional wrenches. However, the actuation wrench can be selected as a wrench in $\mathcal{U}_{\text {Legk }}$ but not in $\mathcal{W}_{\text {Legk }}$.

By locking the actuated parallelogram joint of the $k$ th leg of the IRSBot-2, the actuation wrench of the leg is a screw reciprocal to twists $\hat{\mathcal{E}}_{0 k}$ and $\hat{\mathcal{E}}_{\infty k}$, which does not belong to $\mathcal{W}_{\text {Legk }}$. Accordingly, it is a pure force passing through point $P_{k}$ and along the vector $\mathbf{h}_{k}$ normal to $\mathbf{f}_{1 k} \times \mathbf{f}_{2 k}$ (see Fig. 5). As a result, the actuation wrench system of the robot can be written as:

$$
\mathcal{W}_{I R S}^{a}=\operatorname{span}\left(\hat{\mathcal{F}}_{I}^{a}, \hat{\mathcal{F}}_{I I}^{a}\right)
$$

with $\hat{\mathcal{F}}_{k}^{a}=\left(\mathbf{h}_{k}, \mathbf{r}_{P k} \times \mathbf{h}_{k}\right),(k=I, I I)$. In a non-actuation singular configuration, the legs of the IRSBot-2 apply two linearly independent actuation forces to its end-effector.

\subsection{Constraint singularity conditions of the IRSBot-2}

The IRSBot-2 reaches a constraint singularity when its constraint wrench system $\mathcal{W}_{I R S}^{c}$ defined by Eq. (23) degenerates, namely, when the dimension of $\mathcal{W}_{I R S}^{c}$ is lower than four. $\mathcal{W}_{I R S}^{c}$ is spanned by one pure force and five moments, so its dimension is exactly the number of independent moments plus one. Therefore, it is apparent that $\mathcal{W}_{I R S}^{c}$ degenerates if and only if:

Condition 1: the four moments $\hat{\mathcal{M}}_{1 I}, \hat{\mathcal{M}}_{2 I}, \hat{\mathcal{M}}_{1 I I}$ and $\hat{\mathcal{M}}_{2 I I}$ span a system of dimension one; and/or

Condition 2: the five moments $\hat{\mathcal{M}}_{1 I}, \hat{\mathcal{M}}_{2 I}, \hat{\mathcal{M}}_{1 I I}, \hat{\mathcal{M}}_{2 I I}$ and $\hat{\mathcal{M}}_{I I I}$ span a system of dimension lower than three. 
From Eq. (11), Condition 1 occurs when vectors $\mathbf{n}_{1 I}, \mathbf{n}_{1 I I}, \mathbf{n}_{2 I}$ and $\mathbf{n}_{2 I I}$ that take the following expressions are parallel:

$$
\begin{aligned}
\mathbf{n}_{1 I} & =\left(\cos ^{2} \beta \cos \theta_{I}, \cos \beta \sin \beta \cos \theta_{I},-\sin \theta_{I}\right) \\
\mathbf{n}_{2 I} & =\left(\cos ^{2} \beta \cos \theta_{I},-\cos \beta \sin \beta \cos \theta_{I},-\sin \theta_{I}\right) \\
\mathbf{n}_{1 I I} & =\left(\cos ^{2} \beta \cos \theta_{I I},-\cos \beta \sin \beta \cos \theta_{I I},-\sin \theta_{I I}\right) \\
\mathbf{n}_{2 I I} & =\left(\cos ^{2} \beta \cos \theta_{I I}, \cos \beta \sin \beta \cos \theta_{I I},-\sin \theta_{I I}\right)
\end{aligned}
$$

where angles $\beta, \theta_{I}$ and $\theta_{I I}$ are shown in Figs. 3 and 4.

Practically, Condition 1 holds when the four UU planes are parallel ${ }^{2}$. From Eqs. (29a)-(d), it is apparent that $\mathbf{n}_{1 I}, \mathbf{n}_{1 I I}, \mathbf{n}_{2 I}$ and $\mathbf{n}_{2 I I}$ are parallel if and only if:

$$
\cos \theta_{I}=\cos \theta_{I I}=0
$$

As a matter of fact, Condition 1 holds if and only if the four UU planes are normal to vector $\mathbf{z}_{0}$ due to the symmetry of the manipulator with respect to plane $\mathbf{x}_{0} O \mathbf{z}_{0}$ and the bounds on angle $\beta$, i.e., $0<\beta<\pi / 2$.

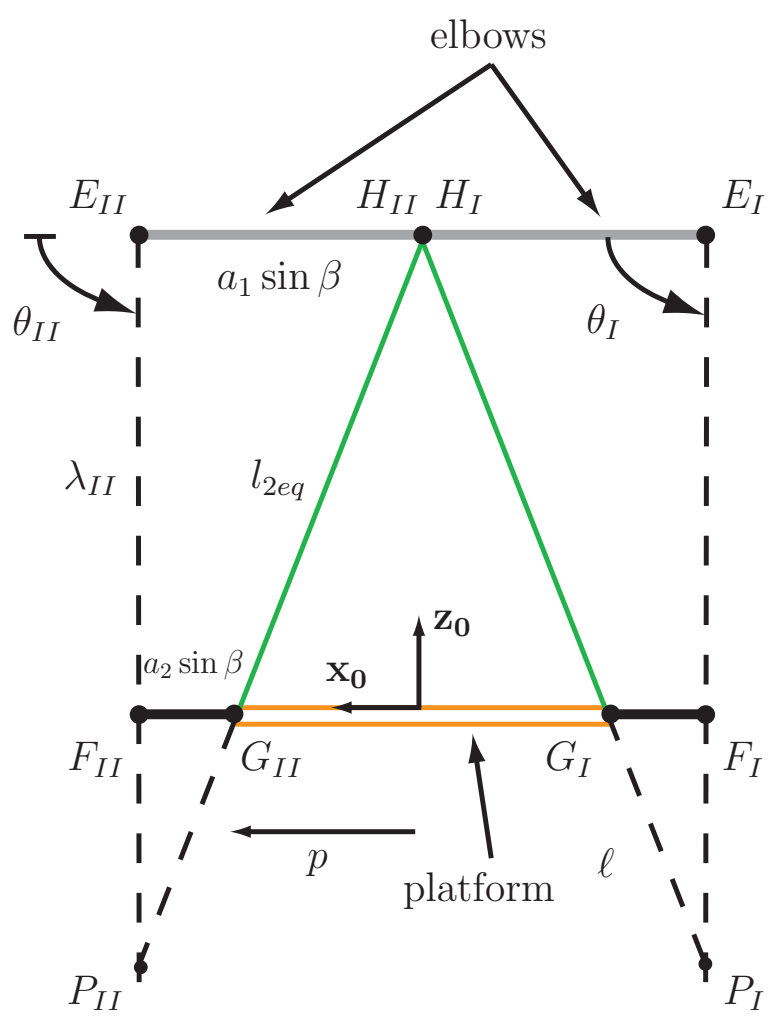

(a) $\theta_{I}=\theta_{I I}=\pi / 2$

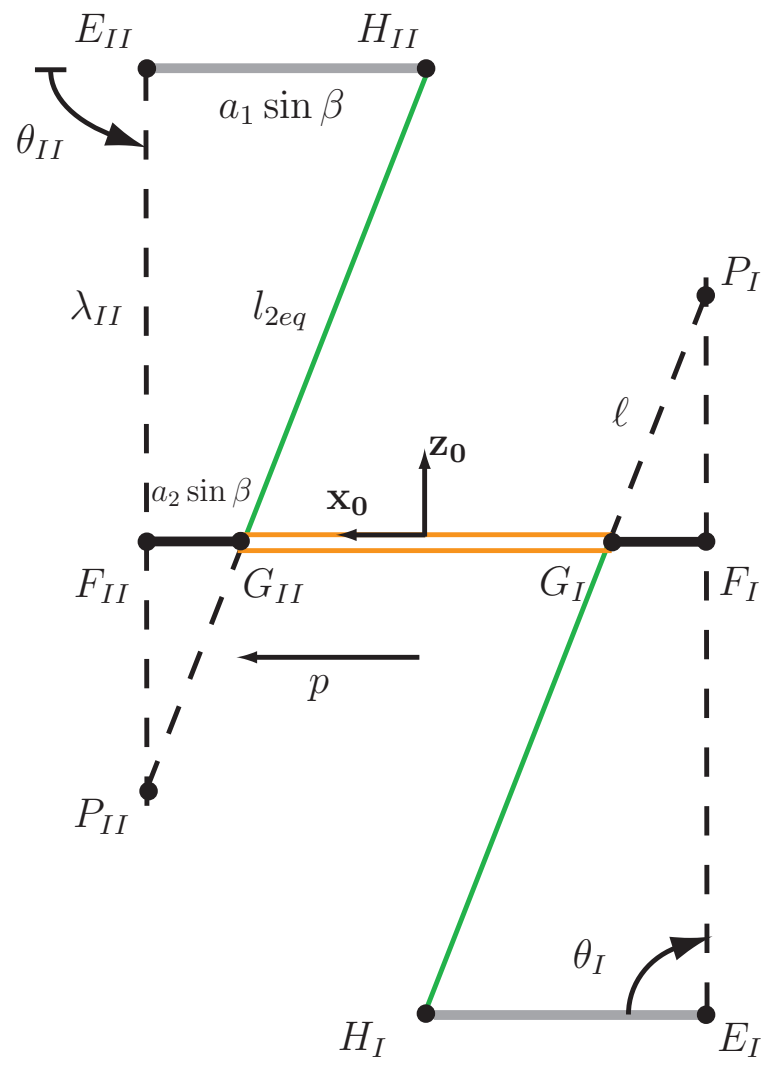

(b) $\theta_{I}=-\pi / 2$ and $\theta_{I I}=\pi / 2$

Figure 8: Constraint singular configurations of the distal loop satisfying Condition 1 (Projection of the distal loop onto the plane $\left(\mathbf{x}_{0} O \mathbf{z}_{0}\right)$ )

\footnotetext{
${ }^{2} \mathrm{UU}$ planes denote the planes including the universal joint axes for each sub-chain $j k$
} 
Figures 8(a)-(b) illustrate the two constraint singular configurations of the distal loop associated with Condition 1.

For the configuration depicted in Fig. 8(a), the directions of the five moments $\mathcal{M}_{1 I}, \mathcal{M}_{1 I I}, \mathcal{M}_{2 I}$, $\mathcal{M}_{2 I I}$ and $\mathcal{M}_{I I I}$ are parallel to $\mathbf{z}_{0}$. As a consequence, the gained motions in this configuration are two infinitesimal rotations about axes lying in the horizontal plane passing through points $P_{1}$ and $P_{2}$. However, this configuration cannot be reached without any collision of the elbows of the IRSBot-2 when the radius $p$ of the platform is smaller than $\left(a_{1}-a_{2}\right) \sin \beta$ (see Fig. 3 for the parameterization).

For the configuration shown in Fig. 8(b), $\theta_{I}=-\pi / 2$ and $\theta_{I I}=\pi / 2$. Therefore, the directions of the five moments $\mathcal{M}_{1 I}, \mathcal{M}_{1 I I}, \mathcal{M}_{2 I}, \mathcal{M}_{2 I I}$ and $\mathcal{M}_{I I I}$ are not parallel, but lie in the plane $\left(\mathbf{x}_{0} O \mathbf{z}_{0}\right)$. As a result, the gained motion of the moving-platform is an infinitesimal rotation about the axis passing through point $P_{I}$ and of unit vector $\mathbf{y}_{0}$. Note that Sec. 6 introduces a methodology to determine the design parameters of the proximal modules that prevent the IRSBot-2 from reaching such singular configurations.

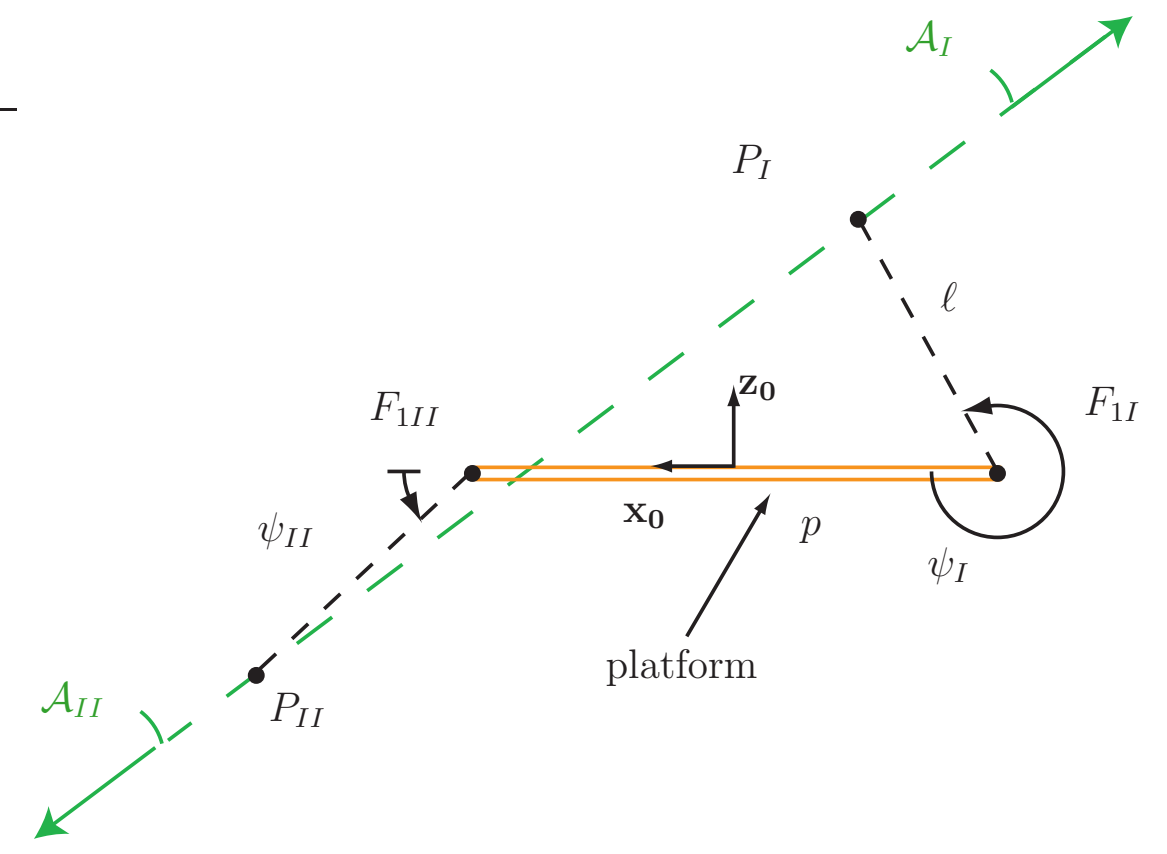

Figure 9: Constraint singular configurations of the distal loop satisfying Condition 2

From Eqs. (11) and (22), Condition 2 occurs if and only if Condition 1 is satisfied and/or vectors $\mathbf{n}_{1 I}, \mathbf{n}_{1 I I}, \mathbf{n}_{2 I}, \mathbf{n}_{2 I I}$ and $\mathbf{y}_{0} \times \overrightarrow{M_{I} M_{I I}}$ have a common normal. The latter condition happens when vectors $\mathbf{n}_{1 I} \times \mathbf{n}_{2 I}, \mathbf{n}_{1 I I} \times \mathbf{n}_{2 I I}$ and $\overrightarrow{P_{I} P_{I I}}$ that take the following expressions are parallel.

$$
\begin{aligned}
\mathbf{n}_{1 I} \times \mathbf{n}_{2 I} & =\left(\sin \theta_{I}, 0, \cos ^{2} \beta \cos \theta_{I}\right) \\
\mathbf{n}_{1 I I} \times \mathbf{n}_{2 I I} & =\left(\sin \theta_{I I}, 0, \cos ^{2} \beta \cos \theta_{I I}\right) \\
\overrightarrow{P_{I} P_{I I}} & =\left(x_{P_{I I}}-x_{P_{I}}, 0, z_{P_{I I}}-z_{P_{I}}\right)
\end{aligned}
$$

As a consequence, $\hat{\mathcal{M}}_{1 I}, \hat{\mathcal{M}}_{2 I}, \hat{\mathcal{M}}_{1 I I}, \hat{\mathcal{M}}_{2 I I}$ and $\hat{\mathcal{M}}_{I I I}$ span a system of dimension equal to two. From Eqs. (31a)-(c), it is apparent that vectors $\mathbf{n}_{1 I} \times \mathbf{n}_{2 I}, \mathbf{n}_{1 I I} \times \mathbf{n}_{2 I I}$ and $\overrightarrow{P_{I} P_{I I}}$ are parallel when 
the axes $\mathcal{A}_{I}$ and $\mathcal{A}_{I I}$, shown in Fig. 7, coincide, namely,

$$
\theta_{I}=\theta_{I I}+p \pi, \quad p=0,1
$$

and

$$
\left(x_{P_{I I}}-x_{P_{I}}\right) \cos ^{2} \beta \cos \theta_{I I}-\left(z_{P_{I I}}-z_{P_{I}}\right) \sin \theta_{I I}=0
$$

where $x_{P_{k}}$ and $z_{P_{k}}$ are the $x$ - and $z$-Cartesian coordinates of point $P_{k}$ expressed in the robot base frame $(k=I, I I)$.

Geometrically, Condition 2 occurs when the four UU planes intersect the same straight line, namely, the line passing through points $P_{I}$ and $P_{I I}$.

Figure 9 represents a configuration of the distal loop of the IRSBot-2 satisfying Condition 2 . The relation between Eq. (32a), Eq. (32b) and the design parameters of the distal modules is difficult to grasp. Therefore, Sec. 4 deals with a detailed analysis of the constraint singularities of the distal loop corresponding to Condition 2 with regard to its design parameters.

\section{Constraint Singularity Analysis of the IRSBot-2 in its Distal Parameter Space}

This section aims to find the sets of distal design parameters $P_{d}=\left\{a_{1}, a_{2}, \beta, p, l_{2 e q}\right\}$ that allow the IRSBot-2 to reach some constraint singularities. Note that the foregoing five design parameters are shown in Fig. 3. $a_{1}, a_{2}, l_{2 e q}$ and $\lambda_{k}$ are the lengths of segments $E_{k} E_{1 k}, F_{k} F_{1 k}, H_{k} G_{k}$ and $E_{k} F_{k}$, respectively. $p$ is the radius of the moving-platform. The definition domains of $a_{1}, a_{2}, \beta, p, l_{2 e q}$ and $\lambda_{k}$ are ]0, $+\infty[] 0,,+\infty[] 0,, \pi / 2[] 0,,+\infty[] 0,,+\infty[$ and $] 0,+\infty\left[\right.$, respectively, with $a_{1}>a_{2}$.

Let $\mathcal{D}_{d}$ denote the definition domain of $P_{d}$ and let $\mathcal{D}_{r}$ be the definition domain of $\left\{a_{1}, a_{2}, \beta, p\right\}$.

The Cartesian coordinates of vector $\overrightarrow{P_{I} P_{I I}}$ shown in Fig. 9 are expressed by the following equation that highlights the relation between the coordinates of points $P_{I}, P_{I I}$ and angles $\psi_{I}, \psi_{I I}$.

$$
\overrightarrow{P_{I} P_{I I}}=\left[\begin{array}{c}
x_{P_{I I}}-x_{P_{I}} \\
z_{P_{I I}}-z_{P_{I}}
\end{array}\right]=\left[\begin{array}{c}
2 p+\ell\left(\cos \psi_{I I}-\cos \psi_{I}\right) \\
-\ell\left(\sin \psi_{I I}-\sin \psi_{I}\right)
\end{array}\right]
$$

with

$$
\ell=\frac{a_{2} l_{2 e q}}{a_{1}-a_{2}}
$$

Angles $\psi_{I}$ and $\psi_{I I}$ are depicted in Figs. 3 and 4. From the closed-loop $E_{k}-H_{k}-G_{k}-F_{k}(k=I, I I)$, the link between $\lambda_{k}, \theta_{k}$ and $\psi_{k}$ is expressed as follows:

$$
\left[\begin{array}{c}
l_{2 e q} \cos \psi_{k} \\
-l_{2 e q} \sin \psi_{k}
\end{array}\right]=\left[\begin{array}{c}
\lambda_{k} \cos \theta_{k}-\left(a_{1}-a_{2}\right) \sin \beta \\
-\lambda_{k} \sin \theta_{k}
\end{array}\right]
$$


The length $\lambda_{k}$, shown in Fig. 4, is obtained from Eq. (35):

$$
\begin{aligned}
\lambda_{k}^{2} & =l_{2 e q}^{2}+\left(a_{1}-a_{2}\right)^{2} \sin ^{2} \beta+2(-1)^{k+1} l_{2 e q}\left(a_{1}-a_{2}\right) \sin \beta \cos \psi_{k} \\
l_{2 e q}^{2} & =\lambda_{k}^{2}+\left(a_{1}-a_{2}\right)^{2} \sin ^{2} \beta-2(-1)^{k+1} \lambda_{k}\left(a_{1}-a_{2}\right) \sin \beta \cos \theta_{k}
\end{aligned}
$$

Equation (36) gives $\lambda_{k}$ as a function of $\psi_{k} . \lambda_{k}$ is also the root of polynomial (37) whose coefficients depend on variable $\theta_{k}$. The foregoing two formulations are used to simplify the constraint singularity condition defined by Eq. (32b).

The following three cases are analyzed separately in order to end up with a univariate polynomial form of Eq. (32b):

Case I: $\theta_{I}=\theta_{I I}+\pi$ and $\lambda_{I}=\lambda_{I I} \neq 0$

Equation (32b) can be expressed in the following polynomial form with variable $X$ corresponding to $\cos \psi_{I I}$ and $\psi_{I}=\psi_{I I}+\pi$ because Eq. (35), $\theta_{I}=\theta_{I I}+\pi$ and $\lambda_{I}=\lambda_{I I} \neq 0$ :

$$
\begin{aligned}
& Q_{I}:[-1,1] \rightarrow \mathbb{R} \\
& \text { with } \begin{array}{c}
X \quad \\
\left.\left.\qquad a_{1}, a_{2}, \beta, p\right] \in \mathcal{D}_{r}, l_{2 e q} \in\right] 0,+\infty[
\end{array} \\
& \left\{\begin{array}{l}
A_{1}=-l_{2 e q}^{2} \sin ^{2} \beta a_{2} /\left(a_{1}-a_{2}\right) \\
B_{1}=l_{2 e q}\left(1-\sin ^{2} \beta\right)\left(p-a_{2} \sin \beta\right) \\
C_{1}=-p\left(a_{1}-a_{2}\right)\left(1-\sin ^{2} \beta\right) \sin \beta+l_{2 e q}^{2} a_{2} /\left(a_{1}-a_{2}\right)
\end{array}\right.
\end{aligned}
$$

Case II: $\theta_{I}=\theta_{I I}+\pi$ and $\lambda_{I} \neq \lambda_{I I}$

Equation (32b) can be expressed in the following polynomial form with variable $X$ corresponding to $\cos \theta_{I I}$ :

$$
\begin{aligned}
& Q_{I I}:[-1,0[\rightarrow \mathbb{R} \\
& X \quad \mapsto \quad Q_{I I}(X)=A_{2} X^{2}+C_{2} \\
& \text { with } \left.\quad\left[a_{1}, a_{2}, \beta, p\right] \in \mathcal{D}_{r}, l_{2 e q} \in\right]\left(a_{1}-a_{2}\right) \sin \beta|\sin \theta|,\left(a_{1}-a_{2}\right) \sin \beta[ \\
& \left\{\begin{array}{l}
A_{2}=a_{2} \sin ^{3} \beta \\
C_{2}=p\left(1-\sin ^{2} \beta\right)-a_{2} \sin ^{3} \beta
\end{array}\right.
\end{aligned}
$$

For $\lambda_{k}$ to be positive in Eq.(37), $l_{2 e q}$ should be bounded between $\left(a_{1}-a_{2}\right) \sin \beta|\sin \theta|$ and $\left(a_{1}-a_{2}\right) \sin \beta$.

Case III: $\theta_{I}=\theta_{I I}$

Equation (32b) can be expressed in the following polynomial form with variable $X$ corresponding 
to $\cos \theta_{I I}$ :

$$
\begin{aligned}
& Q_{I I I}:[-1,1] \rightarrow \mathbb{R} \\
& X \quad \mapsto \quad Q_{I I I}(X)=A_{3} X^{2}+C_{3} \\
& \text { with } \left.\quad\left[a_{1}, a_{2}, \beta, p\right] \in \mathcal{D}_{r}, l_{2 e q} \in\right]\left(a_{1}-a_{2}\right) \sin \beta,+\infty[ \\
& \left\{\begin{array}{l}
A_{3}=a_{2} \sin ^{3} \beta \\
C_{3}=p\left(1-\sin ^{2} \beta\right)-a_{2} \sin ^{3} \beta
\end{array}\right.
\end{aligned}
$$

The lower bound on $l_{2 e q}$, i.e., $\left(a_{1}-a_{2}\right) \sin \beta$, is obtained from Eq. (37).

As a matter of fact, the IRSBot-2 reaches a constraint singularity as long as one of the univariate polynomials (38), (39) and (40) admits one root at least. As the previous algebraic equations are relatively simple, their solutions can be expressed in a closed form. The set of design parameters $\left\{a_{1}, a_{2}, \beta, p, l_{2 e q}\right\}$ for which the constraint singularities associated with Cases I, II and III can be reached are obtained with a method based on the notion of Discriminant Varieties and Cylindrical Algebraic Decomposition. This method provides a formal decomposition of the five dimensional parameter space through an exactly known algebraic variety. It resorts to Gröbner bases for the solutions of systems of equations and is described in [18]. The tools used to perform the computations are implemented in a Maple library called Siropa ${ }^{3}$. In the following computation, the used algebraic variables are $a_{1}, a_{2}, \sin \beta, p$ and $l_{2 e q}$.

Table 1: Cells of $\mathbb{R}^{5}$ where the distal loop of the IRSBot-2 can reach some constraint singularities

\begin{tabular}{l|l|r|l}
\hline \hline Case I & \multicolumn{4}{l}{} \\
\cline { 2 - 4 } & ]$p_{1}, p_{2}[$ & (]$l_{2 e q_{1}}, l_{2 e q_{2}}[)$ & \\
& ]$p_{2}, p_{3}[$ & (]$l_{2 e q_{1}}, l_{2 e q_{2}}[)$ & \\
& ]$p_{3}, p_{4}[$ & (]$l_{2 e q_{1}}, l_{2 e q_{2}}[)$ & Two singular configs. \\
& ]$p_{11}, a_{12}[,] a_{21}, a_{22}[,] \beta_{1}, \beta_{4}[)$ & (]$l_{2 e q_{2}}, l_{2 e q_{1}}[)$ & \\
& ]$p_{5}, p_{6}[$ & (]$l_{2 e q_{2}}, l_{2 e q_{1}}[)$ & \\
& ]$p_{6}, p_{7}[$ & (]$l_{2 e q_{2}}, l_{2 e q_{1}}[)$ & \\
\cline { 2 - 5 } & ]$p_{3}, p_{4}[$ & (]$l_{2 e q_{3}}, l_{2 e q_{1}}[)$ & Four singular configs. \\
\hline Case II & ]$p_{4}, p_{5}[$ & (]$l_{2 e q_{3}}, l_{2 e q_{2}}[)$ & \\
(]$a_{11}, a_{12}[,] a_{21}, a_{22}[,] \beta_{1}, \beta_{4}[)$ & ]$p_{1}, p_{8}[$ & (]$l_{2 e q_{4}}, l_{2 e q_{2}}[)$ & Two singular configs. \\
\hline Case III & & \\
(]$a_{11}, a_{12}[,] a_{21}, a_{22}[,] \beta_{1}, \beta_{4}[)$ & ]$p_{1}, p_{8}[$ & (]$l_{2 e q_{2}}, l_{2 e q_{5}}[)$ & Four singular configs. \\
\hline \hline
\end{tabular}

Table 1 provides the cells of $\mathbb{R}^{5}$ where the distal loop of the IRSBot- 2 can reach some constraint singularities, namely, where $Q_{I}, Q_{I I}$ or $Q_{I I I}$ admits at least one root. The expressions of the lower and upper bounds of those cells are given in Tab. 2. It is noteworthy that parameters $a_{1}$ and $\beta$ can be chosen independently. However, the lower and upper bounds of intervals associated with parameters $a_{2}, p$ and $l_{2 e q}$ are determined successively and depend on the upstream parameters.

For a better understanding of Tables 1 and 2, a set of design parameters $\left\{a_{1}, a_{2}, \beta, p, l_{2 e q}\right\}$ for

\footnotetext{
${ }^{3}$ http://www.irccyn.ec-nantes.fr/ chablat/SIROPA/files/siropa-mpl.html
} 
Table 2: Formulae describing the boundaries of the cells shown in Tables 1 and 3

\begin{tabular}{l|l}
\hline \hline$a_{11}=0$ & $p_{1}=0$ \\
$a_{12}=+\infty$ & $p_{2}\left(a_{2}, \sin \beta\right)=\frac{1-\sin \beta}{1+\sin \beta} a_{2} \sin \beta$ \\
\hline$a_{21}=0$ & $p_{3}\left(a_{2}, \sin \beta\right)=\frac{1-\sin ^{2} \beta}{1+\sin ^{2} \beta} a_{2} \sin \beta$ \\
$a_{22}=a_{1}$ & $p_{4}\left(a_{2}, \sin \beta\right)=a_{2} \sin \beta$
\end{tabular}

which the distal loop of the IRSBot-2 can reach a constraint singularity corresponding to Case I is determined hereafter.

From Tables 1 and 2, $a_{1}$ and $\beta$ should belong to intervals ]0, $+\infty[$ and $] 0, \pi / 2[$, respectively, and can be chosen independently of the other three design parameters. Therefore, let $a_{1}$ and $\beta$ be equal to $0.2 \mathrm{~m}$ and $\pi / 4$, respectively. $a_{2}$ is set to $0.03 \mathrm{~m}$ as it should be positive but smaller than $a_{1}$ (see Tab. 1). Then, the lower and upper bounds of intervals associated with parameter $p$ can be evaluated up to a precision of $10^{-4} \mathrm{~m}$ :

\begin{tabular}{c|ccccccc}
\hline \hline$p$ & $p_{1}$ & $p_{2}$ & $p_{3}$ & $p_{4}$ & $p_{5}$ & $p_{6}$ & $p_{7}$ \\
\hline & 0 & 0.0036 & 0.0071 & 0.0212 & 0.0637 & 0.1236 & $+\infty$ \\
\hline \hline
\end{tabular}

Let $p$ take a value between $p_{4}$ and $p_{5}$ and be equal to $0.05 \mathrm{~m}$. From Table 1 , the distal loop of the IRSBot-2 may reach two or four constraint singularities depending on the choice of $l_{2 e q}$ as $p$ is between $p_{4}$ and $p_{5}$. As a matter of fact, it can reach two symmetrical singular configurations when $l_{2 e q}$ is between $l_{2 e q_{2}}$ and $l_{2 e q_{1}}$, i.e., $Q_{I}$ has one root, and it can reach four symmetrical singular configurations when $l_{2 e q}$ is between $l_{2 e q_{3}}$ and $l_{2 e q_{2}}$, i.e., $Q_{I}$ has two different roots,. Similarly, the lower and upper bounds of intervals associated with parameter $l_{2 e q}$ can be evaluated up to a precision of $10^{-4} \mathrm{~m}$ :

\begin{tabular}{c|ccc}
\hline \hline$l_{2 e q}$ & $l_{2 e q_{1}}$ & $l_{2 e q_{2}}$ & $l_{2 e q_{3}}$ \\
\hline & 2.833 & 0.1202 & 0.1171 \\
\hline \hline
\end{tabular}

Let $l_{2 e q}$ take a value between $l_{2 e q_{2}}$ and $l_{2 e q_{1}}$ and be equal to $0.2 \mathrm{~m}$.

As a result, the polynomial $Q_{I}$ admits one root $X=-0.7388$ lying in the definition domain for the set of design parameters $P_{d}=\{0.2,0.03, \pi / 4,0.05,0.2\}$. The corresponding constraint 


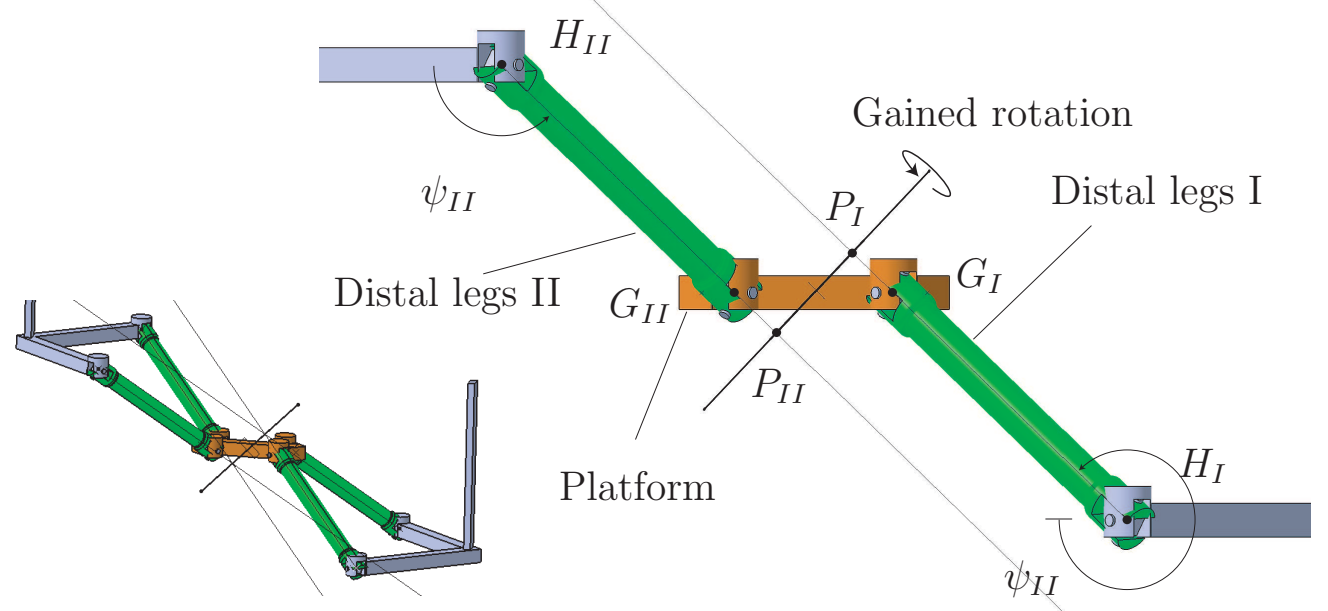

Figure 10: Front view of the distal loop of the IRSBot-2 in a constraint singular configuration corresponding to Case I $\left(\psi_{I I}=\psi_{I}+\pi\right)$

singular configuration of the distal loop of the IRSBot-2 is illustrated in Fig 10. Note that $\psi_{I I}=$ $\arccos (-0.7388)= \pm 2.402 \mathrm{rad}$ for this configuration.

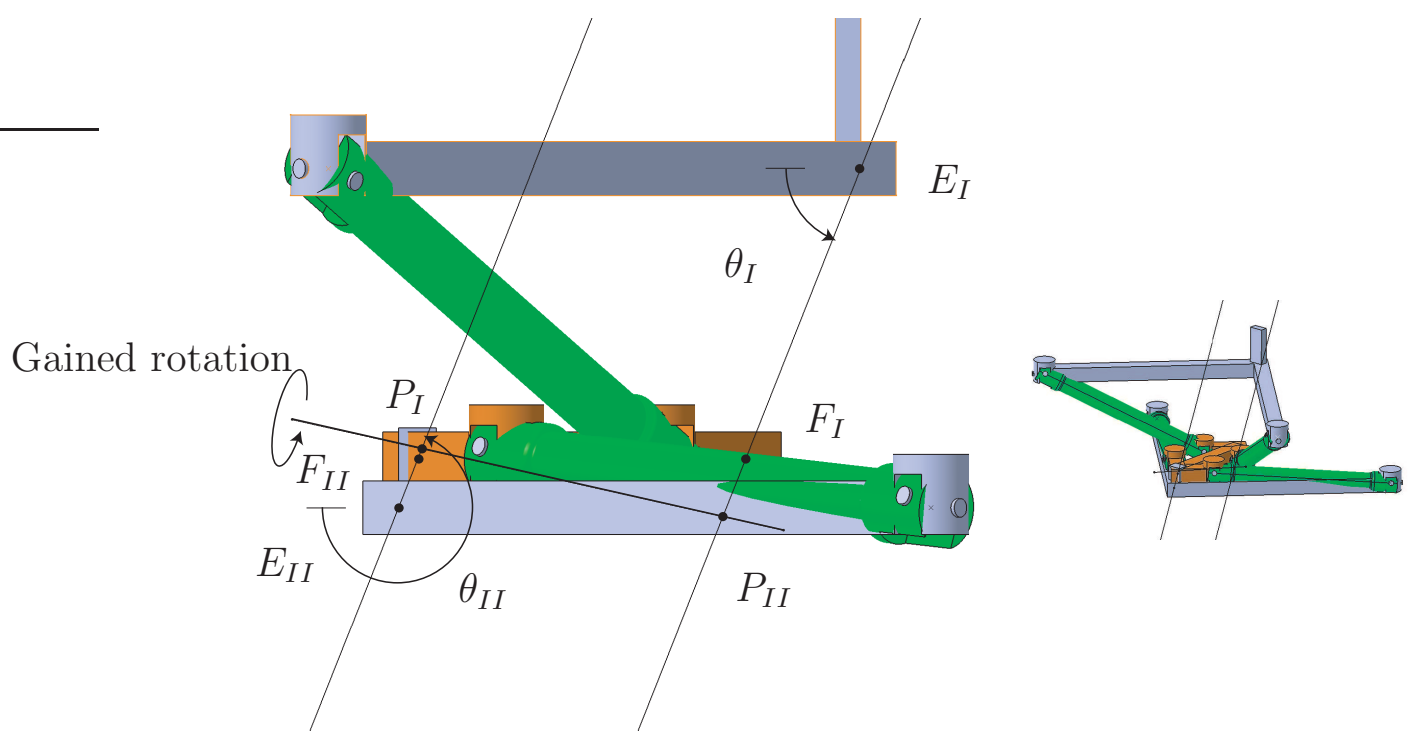

Figure 11: Front view of the distal loop of the IRSBot-2 in a constraint singular configuration corresponding to Case II $\left(\theta_{I I}=\theta_{I}+\pi\right)$

Figures 11 and 12 depict two constraint singular configurations of the distal loop of the IRSBot-2 associated with Cases II and III, respectively. The gained motion of the moving-platform is a rotation about the axis $P_{I} P_{I I}$ shown in Figs. 10, 11 and 12 for the previous three singular configurations. 


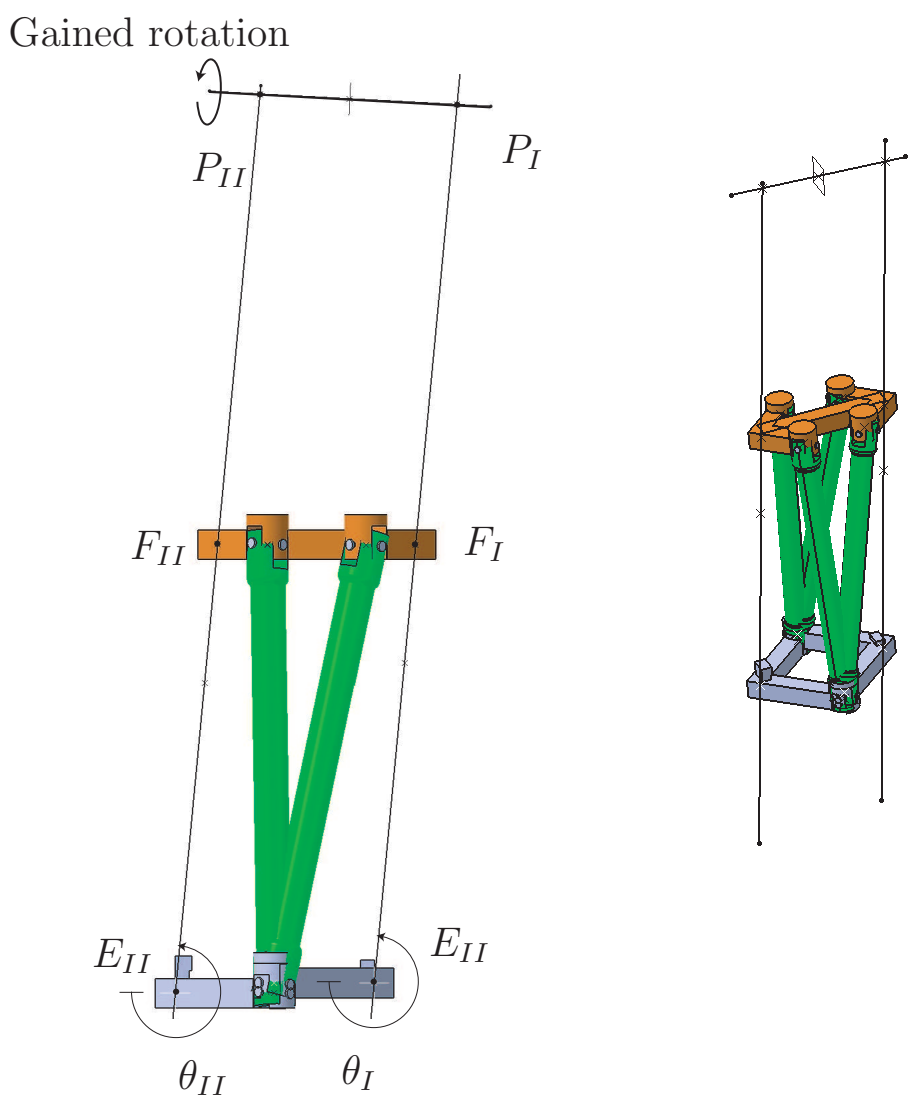

Figure 12: Front view of the distal loop of the IRSBot-2 in a constraint singular configuration corresponding to Case III $\left(\theta_{I I}=\theta_{I}\right)$

\section{Design Parameters for the Distal Loop to be Free of Constraint Singularity}

This section aims to find the sets of design parameters $P_{d}=\left\{a_{1}, a_{2}, \beta, p, l_{2 e q}\right\}$ that prevent the distal loop of the IRSBot-2 from reaching any constraint singularity. It amounts to find the intersection of cells where $Q_{I}, Q_{I I}$ and $Q_{I I I}$ do not have any real root over their mutual domain.

As it is more difficult to obtain the intersection of cells than their union, the cells where the product of $Q_{I}, Q_{I I}$ and $Q_{I I I}$ does not have any real root are searched. From Eqs. (39) and (40), it is apparent that the expressions of $Q_{I I}$ and $Q_{I I I}$ are the same, but their domains are disjointed and complementary because of the bounds of $l_{2 e q}$. Therefore, the sets of design parameters $P_{d}=\left\{a_{1}, a_{2}, \beta, p, l_{2 e q}\right\}$ that prevent the IRSBot-2 from reaching any constraint singularity correspond to the union of cells that do not provide any real root for the following three univariate polynomials:

$$
\begin{aligned}
& Q_{I V}:[-1,1] \rightarrow \mathbb{R} \\
& X \quad \mapsto Q_{I V}(X)=Q_{I} Q_{I I}(X)=\left(A_{1} X^{2}+B_{1} X+C_{1}\right)\left(A_{2}((X-1) / 2)^{2}+C_{2}\right) \\
& \text { with } \left.\quad\left[a_{1}, a_{2}, \beta, p\right] \in \mathcal{D}_{r}, l_{2 e q} \in\right]\left(a_{1}-a_{2}\right) \sin \beta|\sin \theta|,\left(a_{1}-a_{2}\right) \sin \beta[
\end{aligned}
$$




$$
\begin{aligned}
& Q_{V}:[-1,1] \rightarrow \mathbb{R} \\
& X \quad \mapsto Q_{V}(X)=Q_{I} Q_{I I I}(X)=\left(A_{1} X^{2}+B_{1} X+C_{1}\right)\left(A_{3} X^{2}+C_{3}\right) \\
& \text { with } \left.\quad\left[a_{1}, a_{2}, \beta, p\right] \in \mathcal{D}_{r}, l_{2 e q} \in\right]\left(a_{1}-a_{2}\right) \sin \beta,+\infty[
\end{aligned}
$$

$$
\begin{aligned}
Q_{V I}:[-1,1] & \rightarrow \mathbb{R} \\
X & \mapsto Q_{V I}(X)=Q_{I}=\left(A_{1} X^{2}+B_{1} X+C_{1}\right)
\end{aligned}
$$

with $\left.\quad\left[a_{1}, a_{2}, \beta, p\right] \in \mathcal{D}_{r}, l_{2 e q} \in\right] 0,\left(a_{1}-a_{2}\right) \sin \beta\left|\sin \theta_{I I}\right|[$

$A_{1}, B_{1}, C_{1}, A_{2}, C_{2}, A_{3}, C_{3}$ and $\mathcal{D}_{r}$ being defined in Eqs. (38) to (40).

$Q_{I V}$ is the product of $Q_{I}$ and $Q_{I I}$ with a change of variable for $Q_{I I}$ and the most restrictive domain for $l_{2 e q}$ defined in Eq. (39). $Q_{V}$ is the product of $Q_{I}$ and $Q_{I I I}$ with the most restrictive domain for $l_{2 e q}$ defined in Eq. (40). $Q_{V I}$ amounts to $Q_{I}$ with $l_{2 e q}$ being positive but smaller than $\left(a_{1}-a_{2}\right) \sin \beta\left|\sin \theta_{I I}\right|$.

Table 3: Cells of $\mathbb{R}^{5}$ where the distal loop of the IRSBot- 2 cannot reach any constraint singularity with $\underline{\left.a_{1} \in\right] a_{11}, a_{12}\left[\text { and } a_{2} \in\right] a_{21}, a_{22}[}$

\begin{tabular}{l|lll}
\hline \hline$\beta_{1}, \beta_{2}[$ & (]$p_{8}, p_{3}[,] l_{2 e q_{2}}, l_{2 e q_{5}}[),(] p_{3}, p_{4}[,] l_{2 e q_{2}}, l_{2 e q_{5}}[),(] p_{4}, p_{5}[,] l_{2 e q_{1}}, l_{2 e q_{5}}[),(] p_{5}, p_{7}[,] l_{2 e q_{1}}, l_{2 e q_{5}}[)$ \\
{$\left[\beta_{2}, \beta_{3}[\right.$} & (]$p_{8}, p_{4}[,] l_{2 e q_{2}}, l_{2 e q_{5}}[),(] p_{4}, p_{5}[,] l_{2 e q_{1}}, l_{2 e q_{5}}[),(] p_{5}, p_{7}[,] l_{2 e q_{1}}, l_{2 e q_{5}}[)$ \\
{$\left[\beta_{3}, \beta_{4}\right]$} & (]$p_{8}, p_{5}[,] l_{2 e q_{1}}, l_{2 e q_{5}}[),(] p_{5}, p_{7}[,] l_{2 e q_{1}}, l_{2 e q_{5}}[)$ \\
\hline \hline
\end{tabular}

For Eqs. (41) and (43) the interval of $l_{2 e q}$ depends on variable $\theta_{I I}$. It means that the bounds of the cells for which $Q_{I V}$ and $Q_{V I}$ do not have any real root depend on the robot posture. As a consequence, we consider that $l_{2 e q}>\left(a_{1}-a_{2}\right) \sin \beta$ in order to avoid this issue and search for the cells where $Q_{V}$ does not have any real root.

Note that the distal loops free of constraint singularity with $l_{2 e q} \leq\left(a_{1}-a_{2}\right) \sin \beta$ are not interesting in practice as they lead to bulky robots for which the elbows are quite longer than their legs.

$Q_{I V}$ and $Q_{V I}$ are not defined anymore when $l_{2 e q}>\left(a_{1}-a_{2}\right) \sin \beta$. Therefore, the sets of design parameters $P_{d}=\left\{a_{1}, a_{2}, \beta, p, l_{2 e q}\right\}$ for which the distal loop of the IRSBot-2 cannot reach any constraint singularity are expressed in Table 3 .

As an illustrative example, let $a_{1}=1, \beta=\beta_{2}=\arcsin (1 / \sqrt{3})$ and $l_{2 e q}>\left(a_{1}-a_{2}\right) \sin \beta$. Therefore, the design space parameters that prevent the distal loop of the IRSBot-2 from reaching any constraint singularity is obtained from the second line of Table 3. Its boundaries are depicted in Fig. 13.

\section{Proximal Parameters for the IRSBot-2 to be Assembled and Free of Constraint Singularity}

In this section, we search for the design parameters of the proximal module for the IRSBot-2 to be assembled without having any constraint singularity. The design parameters of the proximal module are 


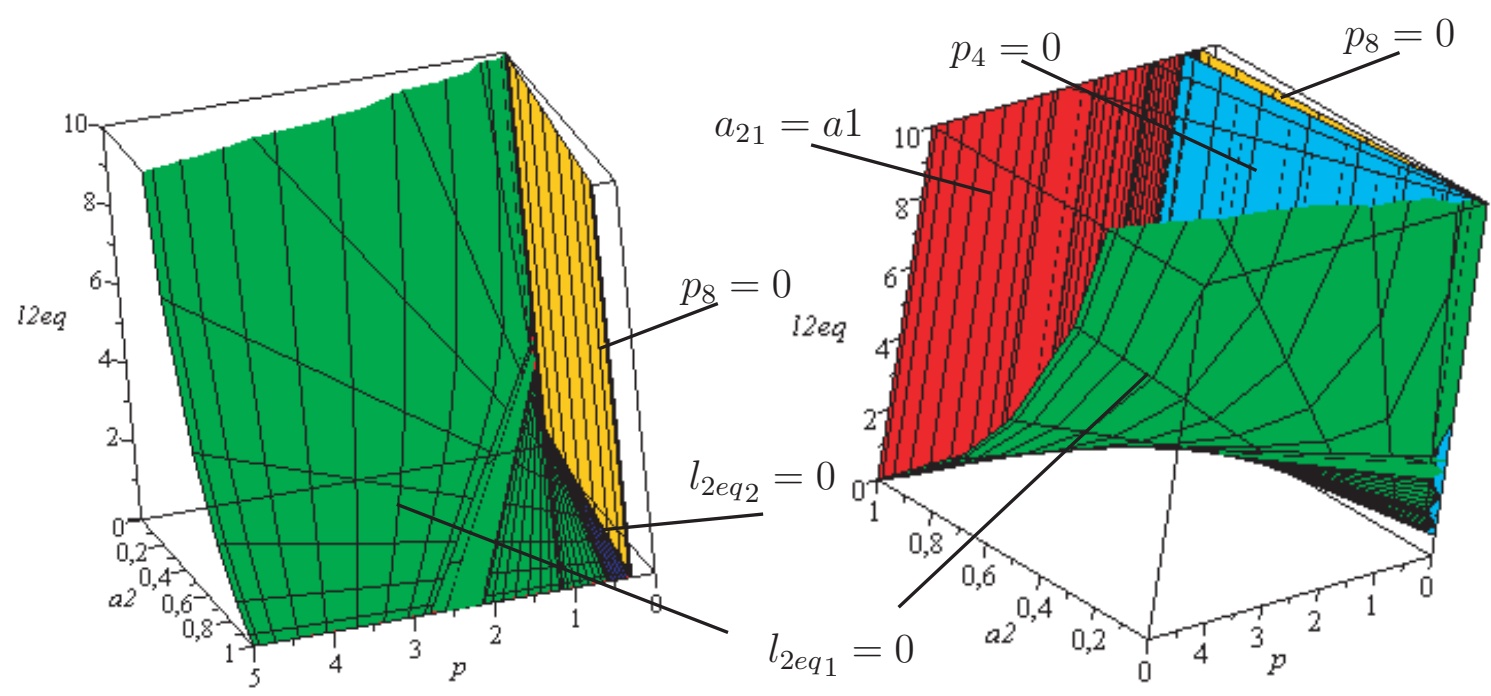

(a)

(b)

Figure 13: Front view (a) and back view (b) of cells where the distal loop of the IRSBot-2 cannot reach any constraint singularity for $a_{1}=1, \beta=\arcsin (1 / \sqrt{3})$ and $l_{2 e q}>\left(a_{1}-a_{2}\right) \sin \beta$

its base radius $b$ and link length $l_{1}$ shown in Fig. 3. The definition domains of $l_{1}$ and $b$ are $] 0,+\infty[$ and ]0, $+\infty$ [, respectively. Let $\mathcal{D}_{p}$ be the definition domain of the set of design parameters $P_{p}=\left\{l_{1}, b\right\}$.

First, the assembly conditions of the IRSBot-2 are analyzed assuming that its distal loop is free of constraint singularity. Then, the conditions on design parameters $l_{1}$ and $b$ for which the IRSBot-2 cannot be assembled in the singular configurations associated with Cases I, II and III are obtained. Finally, a design methodology is proposed to determine the set of design parameters $l_{1}$ and $b$ for the IRSBot-2 to be assembled and free of singularity.

\subsection{First assembly condition of the IRSBot-2}

For a given set of design parameters $P_{d}=\left\{a_{1}, a_{2}, \beta, p, l_{2 e q}\right\}$, the first assembly condition of the IRSBot- 2 with regard to parameters $l_{1}$ and $b$ are obtained by using the assembly condition of the five-bar mechanism [8], namely,

$$
b<l_{1}+l_{2 e q}+a_{1} \sin \beta+p
$$

Moreover, similarly to the condition given in [17] for the five-bar mechanism not to meet any actuation singularity, the IRSBot-2 will not meet any actuation singularity if:

$$
b<-l_{1}+l_{2 e q}+a_{1} \sin \beta+p
$$




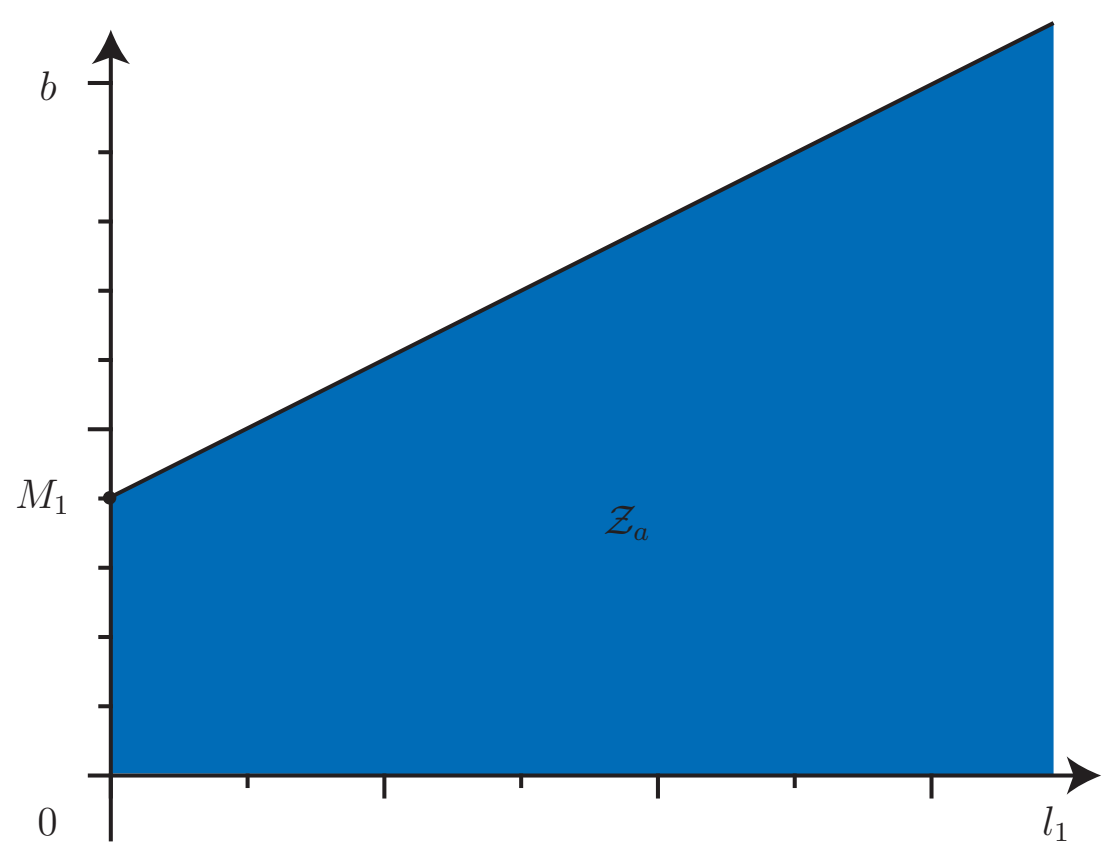

Figure 14: Zone $\mathcal{Z}_{a}$ of the proximal design space $\left\{l_{1}, b\right\}$ where the IRSBot- 2 can be assembled with its distal loop free of constraint singularity

\subsection{Assembly conditions of the IRSBot-2 when its distal loop is free of con- straint singularity}

For a given set $P_{d}$ belonging to Tab. 3 for which the distal loop is free of constraint singularity, inequation (44) can be used to determine the zone $\mathcal{Z}_{a}$ of the proximal design space $\left\{l_{1}, b\right\}$ where the IRSBot- 2 can be assembled. This zone is shown in Fig. 14 and is delimited by the straightline passing through the point $M_{1}$ of coordinates $\left(0, l_{2 e q}+a_{1} \sin \beta+p\right)$ and of slope equal to one. Likewise, the

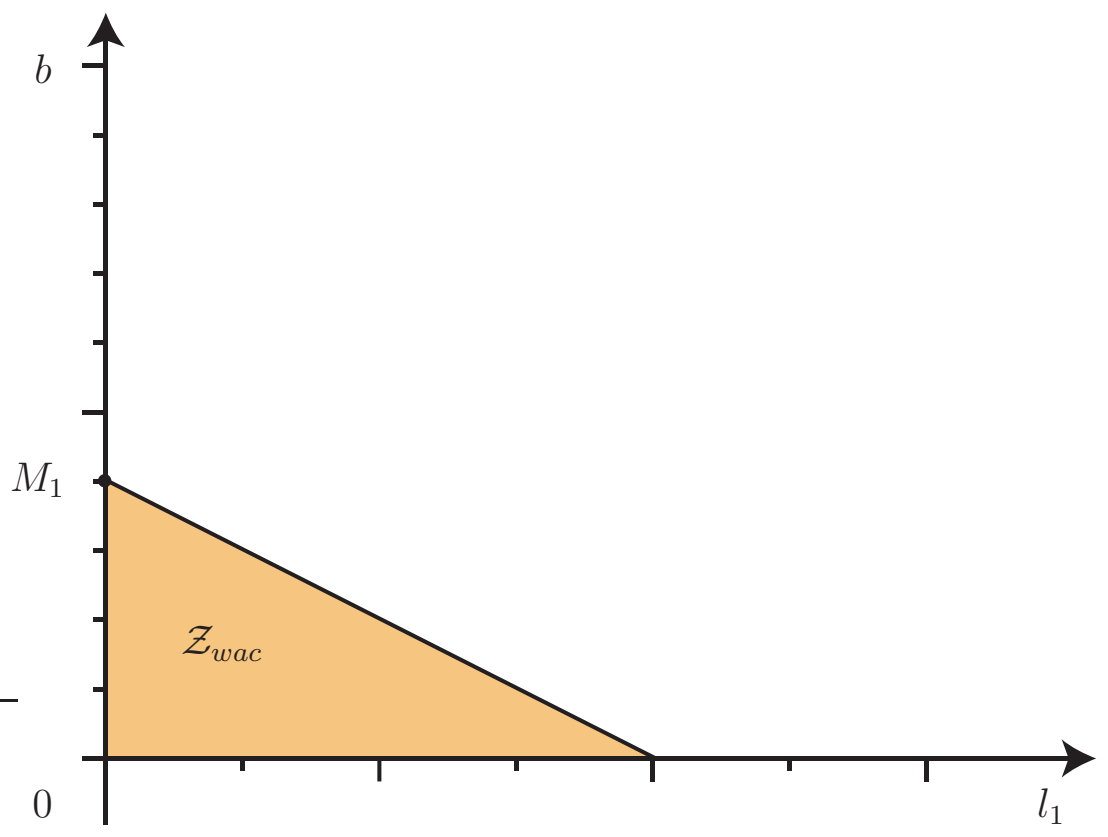

Figure 15: Zone $\mathcal{Z}_{w a c}$ of the proximal design space $\left\{l_{1}, b\right\}$ where the IRSBot-2 can be assembled without any actuation singularity and with its distal loop free of constraint singularity 
zone $\mathcal{Z}_{w a c}$ of the proximal design space, where the IRSBot-2 can be assembled and does not meet any actuation singularity, is obtained thanks to inequation (45). This zone is shown in Fig. 15 and is delimited by the straightline passing through the point $M_{1}$ and of slope equal to minus one.

As a conclusion, the IRSBot-2 can be assembled and neither meets a constraint singularity nor reaches an actuation singularity if the inequations (44) and (45) are satisfied and the design parameters of the distal modules belong to the cells expressed in Table 3.

However, those conditions are restrictive. Indeed, the sets of design parameters $P_{p}$ and $P_{d}$ for the IRSBot- 2 to be free of constraint singularity can be enlarged by considering the cases for which the manipulator is free of constraint singularity when its proximal and distal loops are assembled, whereas its distal loop itself may reach some constraint singularities.

\subsection{Conditions on design parameters $l_{1}$ and $b$ for which the IRSBot-2 cannot be assembled in the constraint singular configurations}

The section aims at finding the conditions on the design parameters $l_{1}$ and $b$ that prevent the IRSBot2 from reaching any constraint singularity even if its distal loop itself may reach some constraint singularities.

From Sec. 4, $Q_{I}\left(Q_{I I}, Q_{I I I}\right.$, resp.) admits one root at least when the design parameters associated with the distal module belong to the cells corresponding to Case. I (Case II, Case III, resp.). It means that the singular posture(s) of the distal loop associated with the $\operatorname{root}(\mathrm{s})$ is(are) known.

The conditions on design parameters $l_{1}$ and $b$ for which the IRSBot- 2 cannot be assembled in the singular configurations associated with Cases I, II and III are obtained hereafter.

Case I: $\theta_{I}=\theta_{I I}+\pi$ and $\lambda_{I}=\lambda_{I I} \neq 0$

For Case I, the assembly conditions of the proximal and distal loops are characterized by the loop-closure $A_{k}-B_{k}-E_{k}-H_{k}-G_{k}-P(k=I, I I)$ that is expressed as follows:

$$
\left[\begin{array}{l}
x \\
z
\end{array}\right]=\left[\begin{array}{c}
l_{1} \cos q_{k} \\
-l_{1} \sin q_{k}
\end{array}\right]+\left[\begin{array}{c}
l_{2 e q} \cos \psi_{k} \\
-l_{2 e q} \sin \psi_{k}
\end{array}\right]+\left[\begin{array}{c}
(-1)^{k}\left(b-a_{1} \sin \beta-p\right) \\
0
\end{array}\right], k=I, I I
$$

As a reminder the conditions $\theta_{I}=\theta_{I I}+\pi$ and $\lambda_{I}=\lambda_{I I} \neq 0$ amount to $\psi_{I}=\psi_{I I}+\pi$. By eliminating $q_{k}$ in Eq. (46) with $\psi_{I}=\psi_{I I}+\pi$, we obtain:

$$
\begin{aligned}
& \left(x+\left(l_{2 e q} \cos \psi+b-a_{1} \sin \beta-p\right)\right)^{2}+\left(z-l_{2 e q} \sin \psi\right)^{2}-l_{1}^{2}=0 \\
& \left(x-\left(l_{2 e q} \cos \psi+b-a_{1} \sin \beta-p\right)\right)^{2}+\left(z+l_{2 e q} \sin \psi\right)^{2}-l_{1}^{2}=0
\end{aligned}
$$

with $\psi$ denoting $\psi_{I I}$ for a better clarity of the equations.

From Eqs. (47a)-(b), the following relation between $x$ and $z$ is obtained:

$$
x=\frac{z l_{2 e q} \sin \psi}{b-\sigma_{1}+l_{2 e q} \cos \psi}
$$


with $\sigma_{1}=a_{1} \sin \beta+p$. The following fourth-degree polynomial is obtained by introducing the previous expression of $x$ into Eq. (47b):

$$
X^{2}+\left(l_{2 e q}^{2} \sin ^{2} \psi-l_{1}^{2}+z^{2}\right) X+\left(z l_{2 e q} \sin \psi\right)^{2}=0
$$

with

$$
X \equiv\left(b-\sigma_{1}+l_{2 e q} \cos \psi\right)^{2}
$$

The discriminant $\Delta$ of Eq. (49) can be expressed as:

$$
\Delta=\left(l_{2 e q} \sin \psi-z-l_{1}\right)\left(l_{2 e q} \sin \psi-z+l_{1}\right)\left(l_{2 e q} \sin \psi+z-l_{1}\right)\left(l_{2 e q} \sin \psi+z+l_{1}\right)
$$

From Eq. (51), $\Delta$ is positive if and only if:

$$
-l_{1}+l_{2 e q}|\sin \psi|<z<l_{1}-l_{2 e q}|\sin \psi|
$$

with $l_{1}>l_{2 e q}|\sin \psi|$. Accordingly, there exist four assembly modes between the proximal loop and the distal loop and four relations appear between the design parameter $b$ and the $z$-Cartesian coordinate of the moving-platform for the IRSBot-2 to be assembled, namely,

$$
\begin{aligned}
& b_{1}^{I}(z)=\sigma_{1}-l_{2 e q} \cos \psi-\frac{1}{2}\left(\sqrt{l_{1}^{2}-\left(l_{2 e q} \sin \psi-z\right)^{2}}-\sqrt{l_{1}^{2}-\left(l_{2 e q} \sin \psi+z\right)^{2}}\right) \\
& b_{2}^{I}(z)=\sigma_{1}-l_{2 e q} \cos \psi-\frac{1}{2}\left(\sqrt{l_{1}^{2}-\left(l_{2 e q} \sin \psi-z\right)^{2}}+\sqrt{l_{1}^{2}-\left(l_{2 e q} \sin \psi+z\right)^{2}}\right) \\
& b_{3}^{I}(z)=\sigma_{1}-l_{2 e q} \cos \psi+\frac{1}{2}\left(\sqrt{l_{1}^{2}-\left(l_{2 e q} \sin \psi-z\right)^{2}}-\sqrt{l_{1}^{2}-\left(l_{2 e q} \sin \psi+z\right)^{2}}\right) \\
& b_{4}^{I}(z)=\sigma_{1}-l_{2 e q} \cos \psi+\frac{1}{2}\left(\sqrt{l_{1}^{2}-\left(l_{2 e q} \sin \psi-z\right)^{2}}+\sqrt{l_{1}^{2}-\left(l_{2 e q} \sin \psi+z\right)^{2}}\right)
\end{aligned}
$$

The extrema of functions $b_{i}^{I}(z), i=1, \ldots, 4$, appear when $z=0$ for a given design parameter $l_{1}$, i.e,

$$
\begin{aligned}
& b_{1}^{I}(z=0)=b_{3}^{I}(z=0)=\sigma_{1}-l_{2 e q} \cos \psi \\
& b_{2}^{I}(z=0)=\sigma_{1}-l_{2 e q} \cos \psi-\sqrt{l_{1}^{2}-l_{2 e q}^{2} \sin ^{2} \psi} \\
& b_{4}^{I}(z=0)=\sigma_{1}-l_{2 e q} \cos \psi+\sqrt{l_{1}^{2}-l_{2 e q}^{2} \sin ^{2} \psi}
\end{aligned}
$$

Note that $b_{2}^{I}(z=0)<b_{1}^{I}(z=0), b_{3}^{I}(z=0)<b_{4}^{I}(z=0)$. As a result, the distal loop in a singular configuration associated with Case I and the proximal loop of the IRSBot-2 can be assembled if and only if:

$$
b_{2}^{I}(z=0)<b<b_{4}^{I}(z=0)
$$

It means that the IRSBot-2 will not reach the corresponding constraint singularity if and only if 
inequation (44) holds and

$$
b<a_{1} \sin \beta+p-l_{2 e q} \cos \psi-\sqrt{l_{1}^{2}-l_{2 e q}^{2} \sin ^{2} \psi}
$$

or

$$
b>a_{1} \sin \beta+p-l_{2 e q} \cos \psi+\sqrt{l_{1}^{2}-l_{2 e q}^{2} \sin ^{2} \psi}
$$

with $l_{1}>l_{2 e q}|\sin \psi|$.

Case II: $\theta_{I}=\theta_{I I}+\pi$ and $\lambda_{I} \neq \lambda_{I I}$

For Case II, the assembly conditions of the proximal and distal loops are characterized by the loop-closure $A_{k}-B_{k}-E_{k}-F_{k}-P(k=I, I I)$ that is expressed as follows:

$$
\left[\begin{array}{l}
x \\
z
\end{array}\right]=\left[\begin{array}{c}
l_{1} \cos q_{k} \\
-l_{1} \sin q_{k}
\end{array}\right]+\left[\begin{array}{c}
\lambda_{k} \cos \theta_{k} \\
-\lambda_{k} \sin \theta_{k}
\end{array}\right]+\left[\begin{array}{c}
(-1)^{k}\left(b-a_{2} \sin \beta-p\right) \\
0
\end{array}\right]
$$

Let $\theta$ denote $\theta_{I I}$ for a better clarity of the equations. By eliminating $q_{k}$ in Eq. (58) with $\theta_{I}=\theta_{I I}+\pi$,

$$
\begin{aligned}
\left(x+\lambda_{I} \cos \theta+\left(b-a_{2} \sin \beta-p\right)\right)^{2}+\left(z-\lambda_{I} \sin \theta\right)^{2}-l_{1}^{2} & =0 \\
\left(x-\lambda_{I I} \cos \theta-\left(b-a_{2} \sin \beta-p\right)\right)^{2}+\left(z+\lambda_{I I} \sin \theta\right)^{2}-l_{1}^{2} & =0
\end{aligned}
$$

Similarly to Case I, a fourth-degree polynomial is obtained from Eqs. (59a) and (59b). Its four roots correspond to four assembly modes between the proximal and distal loops of the IRSBot-2. Those four assembly modes are characterized by the following four relations between the design parameter $b$ and the z-coordinate of the moving platform:

$$
\begin{aligned}
& b_{1}^{I I}(z)=\sigma_{2}-\frac{1}{2}\left(\lambda_{I}+\lambda_{I I}\right) \cos \theta-\frac{1}{2}\left(\sqrt{l_{1}^{2}-\left(z+\lambda_{I I} \sin \theta\right)^{2}}-\sqrt{l_{1}^{2}-\left(z-\lambda_{I} \sin \theta\right)^{2}}\right) \\
& b_{2}^{I I}(z)=\sigma_{2}-\frac{1}{2}\left(\lambda_{I}+\lambda_{I I}\right) \cos \theta-\frac{1}{2}\left(\sqrt{l_{1}^{2}-\left(z+\lambda_{I I} \sin \theta\right)^{2}}+\sqrt{l_{1}^{2}-\left(z-\lambda_{I} \sin \theta\right)^{2}}\right) \\
& b_{3}^{I I}(z)=\sigma_{2}-\frac{1}{2}\left(\lambda_{I}+\lambda_{I I}\right) \cos \theta+\frac{1}{2}\left(\sqrt{l_{1}^{2}-\left(z+\lambda_{I I} \sin \theta\right)^{2}}-\sqrt{l_{1}^{2}-\left(z-\lambda_{I} \sin \theta\right)^{2}}\right) \\
& b_{4}^{I I}(z)=\sigma_{2}-\frac{1}{2}\left(\lambda_{I}+\lambda_{I I}\right) \cos \theta+\frac{1}{2}\left(\sqrt{l_{1}^{2}-\left(z+\lambda_{I I} \sin \theta\right)^{2}}+\sqrt{l_{1}^{2}-\left(z-\lambda_{I} \sin \theta\right)^{2}}\right)
\end{aligned}
$$

The extrema of functions $b_{i}^{I I}(z), i=1, \ldots, 4$, appear when $z=z_{I I}=\frac{1}{2}\left(\lambda_{I}-\lambda_{I I}\right) \sin \theta$ for a given design parameter $l_{1}$, namely,

$$
\begin{aligned}
& b_{1}^{I I}\left(z=z_{I I}\right)=b_{3}^{I I}\left(z=z_{I I}\right)=\sigma_{2}-\frac{1}{2}\left(\lambda_{I}+\lambda_{I I}\right) \cos \theta \\
& b_{2}^{I I}\left(z=z_{I I}\right)=\sigma_{2}-\frac{1}{2}\left(\lambda_{I}+\lambda_{I I}\right) \cos \theta+\sqrt{l_{1}^{2}-\left(\frac{1}{2}\left(\lambda_{I}+\lambda_{I I}\right) \sin \theta\right)^{2}} \\
& b_{4}^{I I}\left(z=z_{I I}\right)=\sigma_{2}-\frac{1}{2}\left(\lambda_{I}+\lambda_{I I}\right) \cos \theta-\sqrt{l_{1}^{2}-\left(\frac{1}{2}\left(\lambda_{I}+\lambda_{I I}\right) \sin \theta\right)^{2}}
\end{aligned}
$$


with $\sigma_{2}=a_{2} \sin \beta+p$.

Note that $b_{2}^{I I}\left(z=z_{I I}\right)<b_{1}^{I I}\left(z=z_{I I}\right), b_{3}^{I I}\left(z=z_{I I}\right)<b_{4}^{I I}\left(z=z_{I I}\right)$. As a result, the distal loop in a singular configuration associated with Case II and the proximal loop of the IRSBot-2 can be assembled if and only if:

$$
b_{2}^{I I}\left(z=z_{I I}\right)<b<b_{4}^{I I}\left(z=z_{I I}\right)
$$

It means that the IRSBot-2 will not reach the corresponding constraint singularity if and only if inequation (44) holds and

$$
b<a_{2} \sin \beta+p-\frac{1}{2}\left(\lambda_{I}+\lambda_{I I}\right) \cos \theta-\sqrt{l_{1}^{2}-\left(\frac{1}{2}\left(\lambda_{I}+\lambda_{I I}\right) \sin \theta\right)^{2}}
$$

or

$$
b>a_{2} \sin \beta+p-\frac{1}{2}\left(\lambda_{I}+\lambda_{I I}\right) \cos \theta+\sqrt{l_{1}^{2}-\left(\frac{1}{2}\left(\lambda_{I}+\lambda_{I I}\right) \sin \theta\right)^{2}}
$$

with $l_{1}>\frac{1}{2}\left(\lambda_{I}+\lambda_{I I}\right)|\sin \theta|$.

Case III: $\theta_{I}=\theta_{I I}$

For Case III, the assembly conditions of the proximal and distal loops are characterized by the loop-closure $A_{k}-B_{k}-E_{k}-F_{k}-P(k=I, I I)$ expressed in Eq. (58). By eliminating $q_{k}$ in Eq. (58) with $\theta_{I}=\theta_{I I}=\theta$,

$$
\begin{array}{r}
\left(x-\lambda_{I} \cos \theta+\left(b-a_{2} \sin \beta-p\right)\right)^{2}+\left(z+\lambda_{I} \sin \theta\right)^{2}-l_{1}^{2}=0 \\
\left(x-\lambda_{I I} \cos \theta-\left(b-a_{2} \sin \beta-p\right)\right)^{2}+\left(z+\lambda_{I I} \sin \theta\right)^{2}-l_{1}^{2}=0
\end{array}
$$

Similarly to Cases I and II, a fourth-degree polynomial is obtained from Eqs. (65a) and (65b). Its four roots correspond to four assembly modes between the proximal and distal loops of the IRSBot-2. Those four assembly modes are characterized by the following four relations between the design parameter $b$ and the $\mathrm{z}$-coordinate of the moving platform:

$$
\begin{aligned}
& b_{1}^{I I I}(z)=\sigma_{2}+\frac{1}{2}\left(\lambda_{I}-\lambda_{I I}\right) \cos \theta-\frac{1}{2}\left(\sqrt{l_{1}^{2}-\left(z+\lambda_{I I} \sin \theta\right)^{2}}-\sqrt{l_{1}^{2}-\left(z+\lambda_{I} \sin \theta\right)^{2}}\right) \\
& b_{2}^{I I I}(z)=\sigma_{2}+\frac{1}{2}\left(\lambda_{I}-\lambda_{I I}\right) \cos \theta-\frac{1}{2}\left(\sqrt{l_{1}^{2}-\left(z+\lambda_{I I} \sin \theta\right)^{2}}+\sqrt{l_{1}^{2}-\left(z+\lambda_{I} \sin \theta\right)^{2}}\right) \\
& b_{3}^{I I I}(z)=\sigma_{2}+\frac{1}{2}\left(\lambda_{I}-\lambda_{I I}\right) \cos \theta+\frac{1}{2}\left(\sqrt{l_{1}^{2}-\left(z+\lambda_{I I} \sin \theta\right)^{2}}-\sqrt{l_{1}^{2}-\left(z+\lambda_{I} \sin \theta\right)^{2}}\right) \\
& b_{4}^{I I I}(z)=\sigma_{2}+\frac{1}{2}\left(\lambda_{I}-\lambda_{I I}\right) \cos \theta+\frac{1}{2}\left(\sqrt{l_{1}^{2}-\left(z+\lambda_{I I} \sin \theta\right)^{2}}+\sqrt{l_{1}^{2}-\left(z+\lambda_{I} \sin \theta\right)^{2}}\right)
\end{aligned}
$$

The extrema of functions $b_{i}^{I I I}(z), i=1, \ldots, 4$, appear when $z=z_{I I I}=-\frac{1}{2}\left(\lambda_{I}+\lambda_{I I}\right) \sin \theta$ for 
a given design parameter $l_{1}$ :

$$
\begin{aligned}
& b_{1}^{I I I}\left(z=z_{I I I}\right)=b_{3}^{I I I}\left(z=z_{I I I}\right)=\sigma_{2}+\frac{1}{2}\left(\lambda_{I}-\lambda_{I I}\right) \cos \theta \\
& b_{2}^{I I I}\left(z=z_{I I I}\right)=\sigma_{2}+\frac{1}{2}\left(\lambda_{I}-\lambda_{I I}\right) \cos \theta+\sqrt{l_{1}^{2}-\left(\frac{1}{2}\left(\lambda_{I}-\lambda_{I I}\right) \sin \theta\right)^{2}} \\
& b_{4}^{I I I}\left(z=z_{I I I}\right)=\sigma_{2}+\frac{1}{2}\left(\lambda_{I}-\lambda_{I I}\right) \cos \theta-\sqrt{l_{1}^{2}-\left(\frac{1}{2}\left(\lambda_{I}-\lambda_{I I}\right) \sin \theta\right)^{2}}
\end{aligned}
$$

Note that $b_{2}^{I I I}\left(z=z_{I I I}\right)<b_{1}^{I I I}\left(z=z_{I I I}\right), b_{3}^{I I I}\left(z=z_{I I I}\right)<b_{4}^{I I I}\left(z=z_{I I I}\right)$. As a result, the distal loop in a singular configuration associated with Case III and the proximal loop of the IRSBot-2 can be assembled if and only if:

$$
b_{2}^{I I I}\left(z=z_{I I I}\right)<b<b_{4}^{I I I}\left(z=z_{I I I}\right)
$$

It means that the IRSBot-2 will not reach the corresponding constraint singularity if and only if Ineq. (44) holds and

$$
b<a_{2} \sin \beta+p+\frac{1}{2}\left(\lambda_{I}-\lambda_{I I}\right) \cos \theta-\sqrt{l_{1}^{2}-\left(\frac{1}{2}\left(\lambda_{I}-\lambda_{I I}\right) \sin \theta\right)^{2}}
$$

or

$$
b>a_{2} \sin \beta+p+\frac{1}{2}\left(\lambda_{I}-\lambda_{I I}\right) \cos \theta+\sqrt{l_{1}^{2}-\left(\frac{1}{2}\left(\lambda_{I}-\lambda_{I I}\right) \sin \theta\right)^{2}}
$$

with $l_{1}>\left|\frac{1}{2}\left(\lambda_{I}-\lambda_{I I}\right) \sin \theta\right|$.

\subsection{Methodology for the determination of the set of design parameters $l_{1}$ and $b$ for the IRSBot-2 to be assembled and free of parallel singularity}

This section aims at introducing a methodology to determine the design parameters $l_{1}$ and $b$ of the proximal modules of the IRSBot- 2 for the latter to be assembled and free of parallel singularity.

For a given set of design parameters $P_{d}=\left\{a_{1}, a_{2}, \beta, p, l_{2 e q}\right\}$, Tab. 1 allows us to know whether the distal loop can reach a constraint singularity or not.

If the distal loop cannot reach any constraint singularity, the reader will be referred to Sec. 6.2 to know the sets of design parameters $\left\{l_{1}, b\right\}$ associated with the proximal modules for which the IRSBot-2 can be assembled.

If the distal loop can reach a constraint singularity, the corresponding constraint singularity case (Case I, II and/or III) and constraint singular configuration will be obtained from Tab. 1. The assembly condition (44) and inequations (56), (57), (63), (64), (69) and (70) determine the set of design parameters $\left\{l_{1}, b\right\}$ for the IRSBot- 2 not to be assembled in that Constraint Singular Configuration (CSC), but to be assembled in non-singular configurations.

Accordingly, Fig. 16 illustrates a flowchart for the determination of the set of design parameters $l_{1}$ 


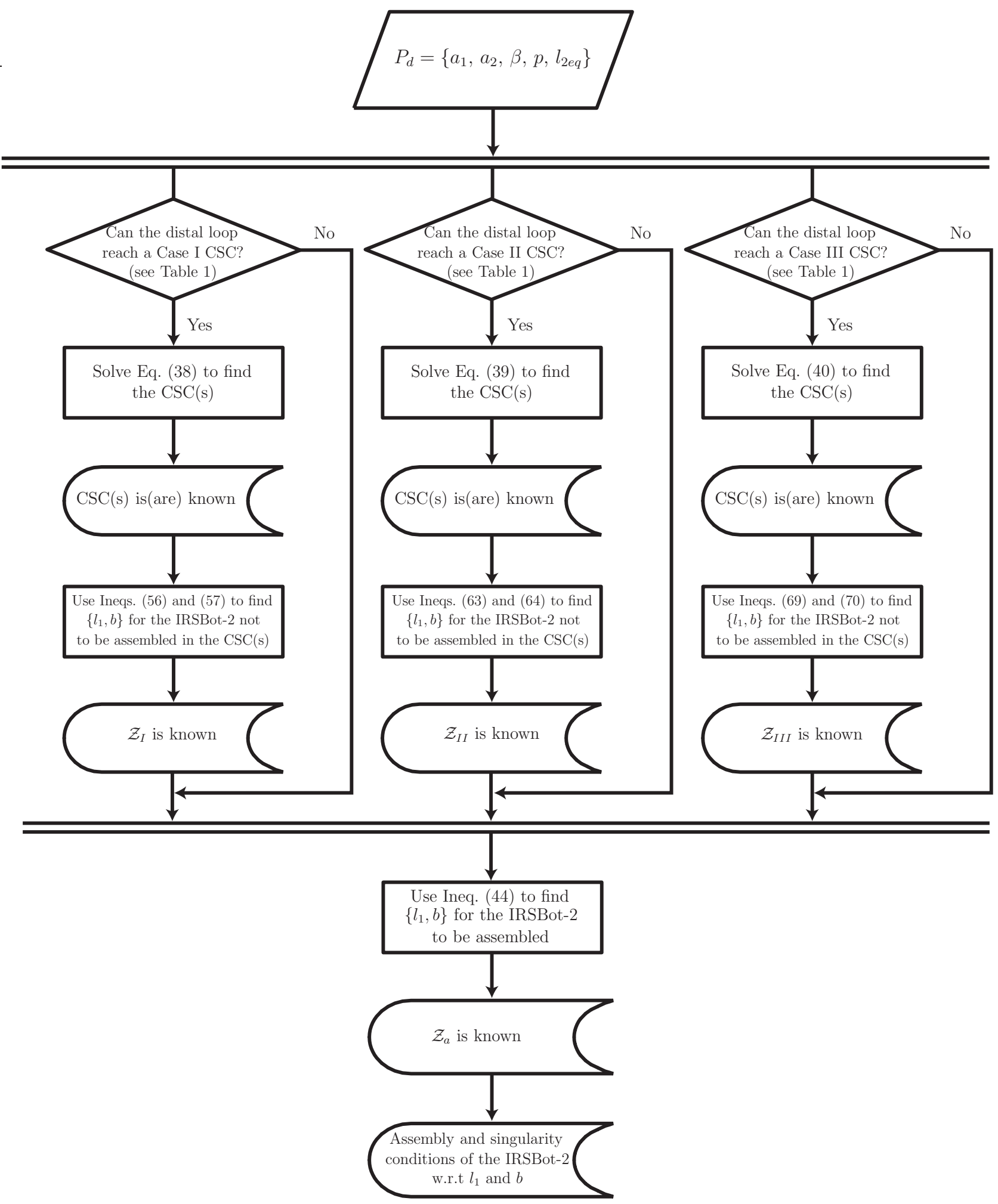

Figure 16: Flowchart for the determination of the set of design parameters $l_{1}$ and $b$ for the IRSBot-2 to be assembled and free of Constraint Singular Configuration (CSC) 
and $b$ for the IRSBot-2 to be assembled and free of parallel singularity.

For a better understanding of the flowchart, let us analyze the IRSBot- 2 for which the design parameters of the distal modules take the following values:

$$
\begin{aligned}
a_{1} & =0.3 \mathrm{~m} \\
a_{2} & =0.05 \mathrm{~m} \\
\beta & =\frac{\pi}{4} \mathrm{rad} \\
p & =0.04 \mathrm{~m} \\
l_{2 e q} & =0.15 \mathrm{~m}
\end{aligned}
$$

From Tab. 1, the distal loop can reach two Case I Constraint Singular Configurations (CSCs), named $C S C_{I 1}$ and $C S C_{I 2}$, and cannot reach any Case II and Case III CSC.

From Eq. (38), the distal loop of the IRSBot-2 reaches the constraint singular configuration $C S C_{I 1}$ when

$$
\cos \psi_{I I}=-0.581
$$

and the constraint singular configuration $\mathrm{CSC}_{I 2}$ when

$$
\cos \psi_{I I}=0.737
$$

From Ineqs. (56) and (57), the IRSBot-2 will not reach $C S C_{I 1}$ if the set $\left\{l_{1}, b\right\}$ belongs to the zone $\mathcal{Z}_{I 1}$ shown in Fig. 17(a) and defined by the following inequations:

$$
\begin{aligned}
& b<0.339-\sqrt{l_{1}^{2}-0.015} \\
& b>0.339+\sqrt{l_{1}^{2}-0.015}
\end{aligned}
$$

From Ineqs. (56) and (57), the IRSBot-2 will not reach $C S C_{I 2}$ if the set $\left\{l_{1}, b\right\}$ belongs to the zone $\mathcal{Z}_{I 2}$ shown in Fig. 17(b) and defined by the following inequations:

$$
\begin{aligned}
& b<0.142-\sqrt{l_{1}^{2}-0.010} \\
& b>0.142+\sqrt{l_{1}^{2}-0.010}
\end{aligned}
$$

As a consequence, the IRSBot- 2 will not reach any constraint singularity if the set $\left\{l_{1}, b\right\}$ belongs to the zone $\mathcal{Z}_{1}$, which is the intersection of $\mathcal{Z}_{I 1}$ and $\mathcal{Z}_{I 2}$ and shown in Fig. 17(c) .

From Ineq. (44), the IRSBot- 2 can be assembled if and only if (iff) the set $\left\{l_{1}, b\right\}$ belongs the zone $\mathcal{Z}_{a}$ illustrated in Fig. 17(d) and characterized by the following inequation:

$$
b<l_{1}+0.402
$$

Therefore, the IRSBot-2 can be assembled and does not reach any constraint singularity iff the set 
$\left\{l_{1}, b\right\}$ belongs to the intersection of $\mathcal{Z}_{a}$ and $\mathcal{Z}_{1}$, named $\mathcal{Z}$ and depicted in Fig. 18(a).

Nevertheless, the IRSBot- 2 can still reach some actuation singularities if the set $\left\{l_{1}, b\right\}$ belongs to $\mathcal{Z}$. From Ineq. (45), the IRSBot- 2 will not meet any actuation singularity if the set $\left\{l_{1}, b\right\}$ belongs to $\mathcal{Z}_{\text {wac }}$ represented in Fig. 15 and defined by the following inequation:

$$
b<-l_{1}+0.402
$$

Finally, the IRSBot-2 can be assembled and cannot reach any parallel singularity, i.e., neither constraint singularity nor actuation singularity, if and only if the set $\left\{l_{1}, b\right\}$ belongs to the zone $\mathcal{Z}_{\text {wps }}$ highlighted in Fig.18(b).

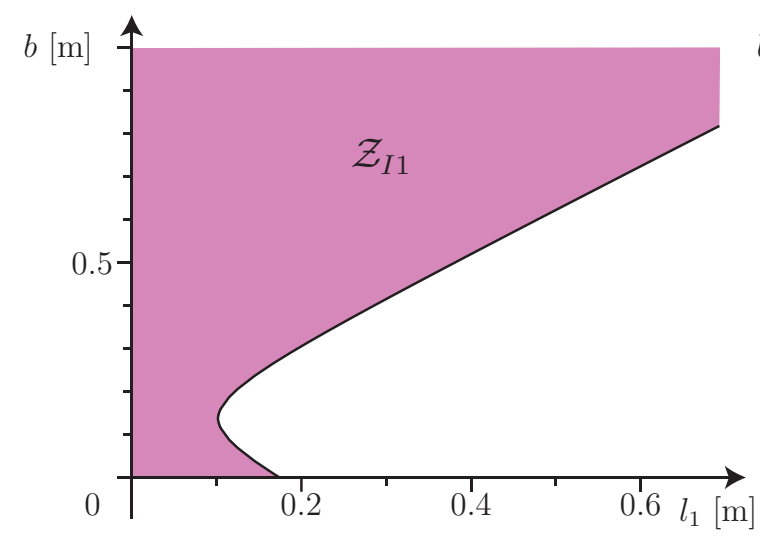

(a)

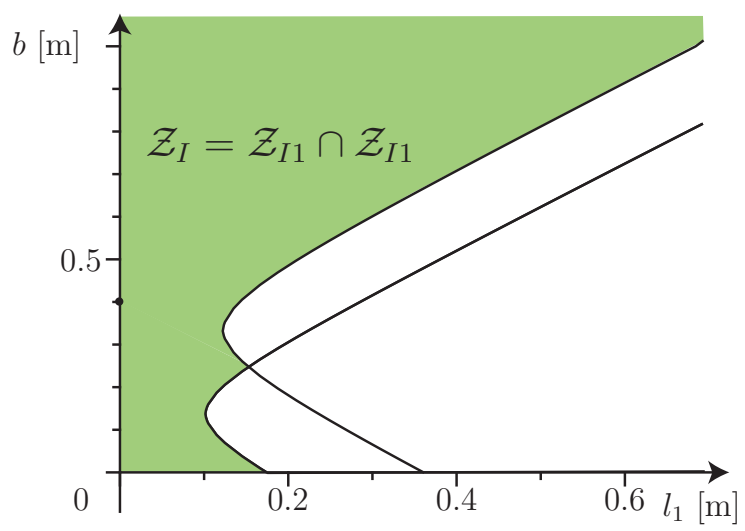

(c)

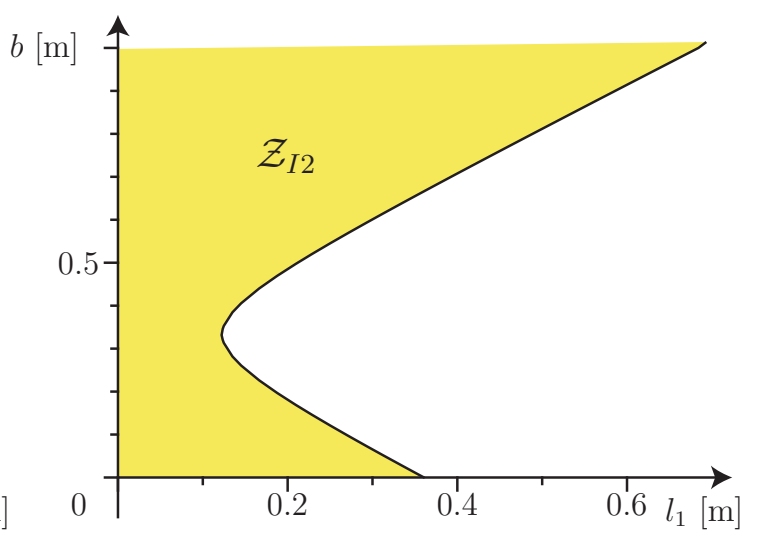

(b)

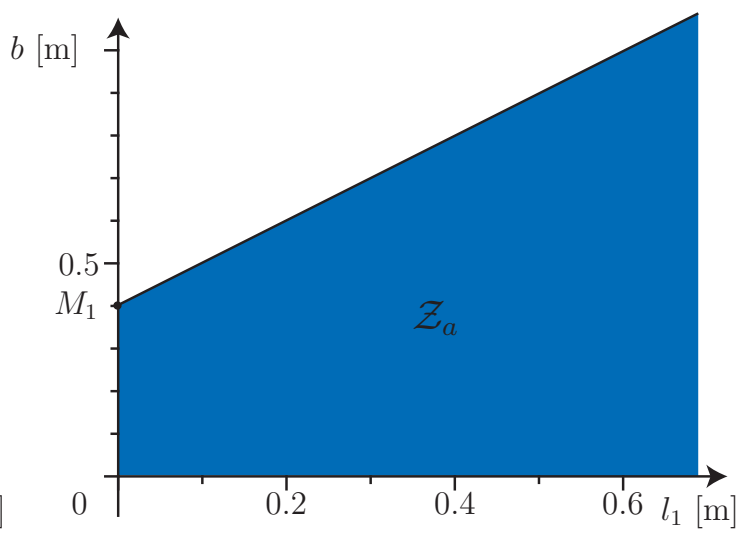

(d)

Figure 17: Assembly and singularity conditions of the IRSBot-2 with regard to design parameters $l_{1}$ and $b$ : (a) $\mathcal{Z}_{I 1}$ : zone of the design space $\left\{l_{1}, b\right\}$ where the IRSBot- 2 can not reach the constraint singular configuration $C S C_{I 1}$; (b) $\mathcal{Z}_{I 2}$ : zone of the design space $\left\{l_{1}, b\right\}$ where the IRSBot- 2 can not reach the constraint singular configuration $C S C_{I 2}$; (c) $\mathcal{Z}_{1}$ : zone of the design space $\left\{l_{1}, b\right\}$ where the IRSBot- 2 can not reach any constraint singular configuration; (d) $\mathcal{Z}_{a}$ : zone of the design space $\left\{l_{1}, b\right\}$ where the IRSBot-2 can be assembled 


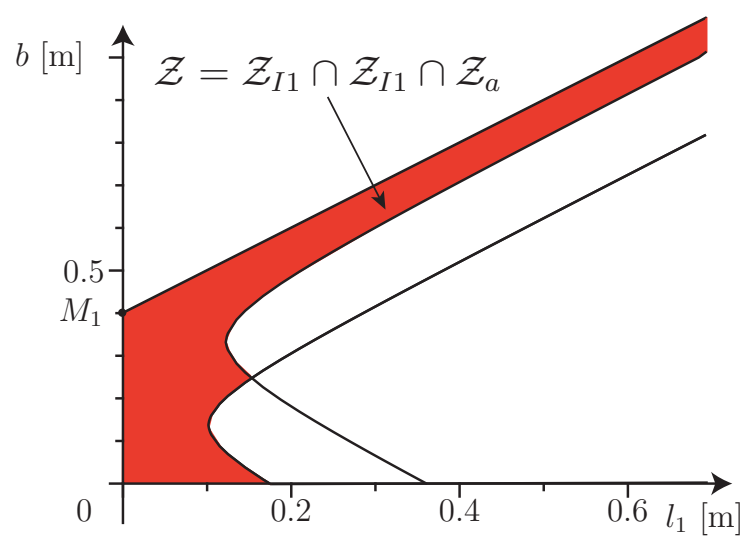

(a)

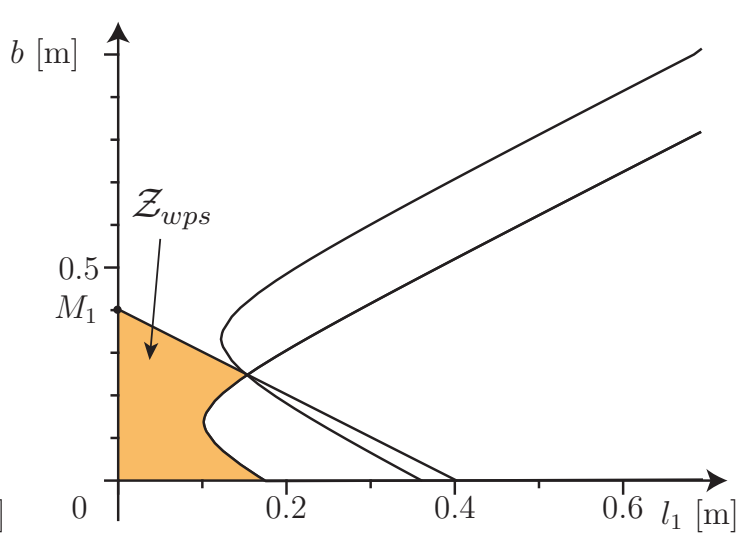

(b)

Figure 18: Assembly and singularity conditions of the IRSBot-2 with regard to design parameters $l_{1}$ and $b$ : (a) $\mathcal{Z}$ : zone of the design space $\left\{l_{1}, b\right\}$ where the IRSBot-2 can be assembled, does not reach any constraint singularity but can reach some actuation singularities; (b) $\mathcal{Z}_{w p s}$ : zone of the design space $\left\{l_{1}, b\right\}$ where the IRSBot- 2 can be assembled and is free of parallel singularity 


\section{Conclusions}

In this paper, a deep analysis was carried out to determine the sets of design parameters of the IRSBot-2 that prevent it from reaching any parallel singularity. From [10], this two-dof manipulator is lighter than the Par2 and the five-bar mechanism while being stiffer than the latter. To the best of our knowledge, such an analysis had never been performed before and is very helpful for the robot designer. Indeed, it allows the robot designer to select the design parameters of the manipulator in such a way that the manipulator cannot reach any parallel singularity.

First, the constraint wrench system $\mathcal{W}_{I R S}^{c}$ and the actuation wrench system $\mathcal{W}_{I R S}^{a}$ of the IRSBot-2 were expressed by using the screw theory. The parallel singularity conditions of the IRSBot- 2 were obtained by analyzing the degeneracy conditions of $\mathcal{W}_{I R S}^{c}$ and $\mathcal{W}_{I R S}^{a}$.

On the one hand, the IRSBot-2 reaches an actuation singular configuration when the actuation forces applied by its two legs on the moving-platform are linearly dependent. On the other hand, two constraint singularity conditions were highlighted and expressed in a vector form and analytically for the IRSBot-2 based on the degeneracy conditions of $\mathcal{W}_{I R S}^{c}$. Three singularity cases were derived from the second constraint singularity condition.

The sets of design parameters associated with the distal modules for the IRSBot- 2 to be able to reach some constraint singularities were obtained with a method based on the notion of Discriminant Varieties and Cylindrical Algebraic Decomposition. This method provided the cells of $\mathbb{R}^{5}$ where the distal loop of the IRSBot-2 can reach some constraint singularities. The lower and upper bounds of those cells were expressed analytically. Three constraint singular configurations of the distal loop of the IRSBot-2 were represented as illustrative examples.

Likewise, a deep analysis was carried out in order to determine the set of design parameters of the distal modules that prevent the distal loop of the IRSBot-2 from reaching any constraint singularity.

Finally, a design methodology was proposed to determine the set of design parameters associated with the proximal modules for the IRSBot- 2 to be assembled and free of actuation singularity and constraint singularity, namely, free of parallel singularity.

The contributions of this paper will be used for the design optimization of the IRSBot-2 later on. The type-synthesis of novel two degrees of freedom translational parallel manipulators with spatial limbs is also part of the future work.

\section{Acknowledgment}

This work was conducted with the support of the French National Research Agency (Project ANR2011-BS3-006-01-ARROW). The authors also thank Damien Chablat for his great help with the Siropa Maple library. 


\section{References}

[1] Amine, S.: Lower-mobility parallel manipulators: Geometrical analysis, singularities and conceptual design. Ph.D. thesis, Ecole Centrale Nantes (2012)

[2] Amine, S., Tale-Masouleh, M., Caro, S., Wenger, P., Gosselin, C.: Singularity Analysis of the 4-RUU Parallel Manipulator Based on Grassmann-Cayley Algebra and Grassmann Geometry. In: International Design Engineering Technical Conferences (IDETC), no. 48226 in DETC2011. Washington, DC, USA (2011)

[3] Amine, S., Tale-Masouleh, M., Caro, S., Wenger, P., Gosselin, C.: Singularity Conditions of 3T1R Parallel Manipulators with Identical Limb Structures. ASME Journal of Mechanisms and Robotics 4(1), 11-1 - 11-11 (2012)

[4] Angeles, J., Caro, S., Khan, W., Morozov, A.: Kinetostatic design of an innovative schonfliesmotion generator. Proceedings of IMechE Part C: Journal of Mechanical Engineering Science 220(7), 935-943 (2006)

[5] Brogardh, T.: Device for relative movement of two elements. Patent US 6301988 B1 (2001)

[6] Caro, S., Khan, W.A., Pasini, D., Angeles, J.: The rule-based conceptual design of the architecture of serial schönflies-motion generators. Mechanism and Machine Theory 45(2), 251-260 (2010)

[7] Clavel, R.: Device for the movement and positioning of an element in space. Patent US 4976582 (1990)

[8] Damien, C.: Domaines d'unicité et parcourabilité pour les manipulateurs pleinement parallèles. Ph.D. thesis, Ecole Centrale Nantes (1998)

[9] Dimentberg, F.M.: The screw calculus and its applications in mechanics. Tech. rep., Foreign Technology Division, Wright-Paterson Air Force Base (1965)

[10] Germain, C., Briot, S., Glazunov, V., Caro, S., Wenger, P.: Irsbot-2: A novel two-dof parallel robot for high-speed operations. In: Proceedings of the ASME 2011 International Design Engineering Technical Conferences \& Computers and Information in Engineering Conference. Washington, DC, USA, August, 29-31 (2011)

[11] Huang, T., Li, M., Li, Z., Chetwynd, D., Whitehouse, D.: Planar parallel robot mechanism with two translational degrees of freedom. Patent WO 03055653 A1 (2003)

[12] Hunt, K.H.: Kinematic Geometriy of Mechanisms. Clarendon Press, Oxford (1978)

[13] Kong, X., Gosselin, C.M.: Type synthesis of parallel mechanisms. Springer Verlag (2007)

[14] Krut, S., Nabat, V., Company, O., Pierrot, F.: A high-speed parallel robot for scara motions. In: Robotics and Automation, 2004. Proceedings. ICRA '04. 2004 IEEE International Conference on, vol. 4, pp. 4109 - 4115 (2004) 
[15] Lee, C.C., Hervé, J.M.: On some applications of primitive Schönflies-motion generators. Mechanism and Machine Theory 44(12), 2153-2163 (2009)

[16] Liu, X., Kim, J.: Two novel parallel mechanisms with less than six degrees of freedom and the applications. In: Proc. Workshop on Fundamental Issues and Future Reserch Directions for Parallel Mechanisms and Manipulators, pp. 172-177. Quebec city, Quebec, Canada (2002)

[17] Maille, B.: Etude comparative des mecanismes parallèles, application à l'usinage. Master's thesis, Ecole Centrale Nantes (2000)

[18] Moroz, G., Chablat, D., Wenger, P., Rouiller, F.: Cusp points in the parameter space of rpr2prr parallel manipulator. In: 3-rd European Conference on Mechanism Science, Cluj-Napoca, Romania, Springer, pp. 29-37 (2010)

[19] Nabat, V., Pierrot, F., de la O Rodriguez Mijangos, M., Azcoita Arteche, J.M., Bueno Zabalo, R., Company, O., Florentino Perez De Armentia, K.: High-speed parallel robot with four degrees of freedom. Patent EP 1870214 A1 (2007)

[20] Pierrot, F., Krut, S., Company, O., Nabat, V., Baradat, C., Fernandez, A.S.: Two degree-offreedom parallel manipulator. Patent WO 2009/089916 A1 (2009)

[21] Zlatanov, D., Bonev, I., Gosselin, C.: Constraint singularities of parallel mechanisms. In: Robotics and Automation, 2002. Proceedings. ICRA '02. IEEE International Conference on, vol. 1, pp. 496 - 502 (2002) 